This is a pre-print of the following article: Varvenne, C.; Luque, A.; Curtin, W. A. Acta Mater. 2016, 118, 164-176.. The formal publication is available at http://dx.doi.org/10.1016/j.actamat.2016.07.040

\title{
Theory of Strengthening in fcc High Entropy Alloys
}

\author{
Céline Varvenne ${ }^{\mathrm{a}, 1, *}$, Aitor Luque ${ }^{\mathrm{a}}$, William A. Curtin ${ }^{\mathrm{a}}$ \\ ${ }^{a}$ Laboratory for Multiscale Materials Modelling, Institute of Mechanical Engineering, École Polytechnique Fédérale de \\ Lausanne, Lausanne CH-1015, Switzerland
}

\begin{abstract}
High Entropy Alloys (HEAs) are a new class of random alloys having impressive strength and toughness. Here, a mechanistic, parameter-free, and predictive theory for the temperature-, composition-, and strainrate-dependence of the plastic yield strength of fcc HEAs is presented, validated, and applied to understand recent experiments. To first order, each elemental component in the HEA is considered as a solute embedded in the effective matrix of the surrounding alloy. Strengthening is then mainly achieved due to dislocation interactions with the random local concentration fluctuations around the average composition. The theory is validated against molecular simulations on model Fe-Ni-Cr alloys. Hall-Petch-corrected yield strengths in Ni-Co-Cr-Fe-Mn fcc HEAs are then predicted using only available experimental information, and good quantitative agreement is achieved. The theory demonstrates the origins of the high strength and detailed trends with composition, materials parameters, temperature, thus identifying the key measurable/calculable material properties needed for design and optimization of fcc HEAs, and is a general model for fcc random alloys.
\end{abstract}

Keywords: High Entropy Alloys, Mechanical properties, Solution Strengthening theory, Yield stress, Molecular Simulations

\section{Introduction}

High Entropy Alloys (HEAs) are random solidsolution alloys with many components. For an $N$ component alloy, the nominal composition is $1 / N$ for each component, with the disorder presumed to facilitate fabrication of single phase materials [1, 2, 3. Recently fabricated fcc HEAs have very high tensile strength at low temperatures and high retained strength at elevated temperatures [4, 5]. Furthermore, these fcc HEAs can have high tensile elongation/ductility and exceptional fracture toughness [1, 2, 6. The spectrum of impressive mechanical properties makes this new broad class 7. of structural materials attractive for many applications. However, the physical mechanism(s) of

\footnotetext{
${ }^{*}$ Corresponding author

Email address: varvenne@univ-mrs.fr (Céline Varvenne)

${ }^{1}$ Present address: Centre Interdisciplinaire des Nanosciences de Marseille, Aix-Marseille UniversityCNRS, Campus de Luminy, case 913, Marseille F-13288, France
}

Preprint submitted to Acta Materialia the strengthening of HEA alloys remain unknown 2, 3, 4, 5. More broadly, although the behavior of random alloys has been a major topic in metal physics over the last fifty years, there is no predictive theory for alloy strength with high elemental concentrations.

Here, we develop and validate a mechanistic theory for the yield stress of general fcc random alloys at arbitrary composition, thus including fcc HEAs, and we demonstrate its predictive capability for the well-studied fcc Ni-Co-Fe-Cr-Mn alloys [4, 5. 8. The theory rationalizes experimental results, quantitatively and qualitatively, and identifies the underlying material properties that control strength, thus also serving as a basis for design and optimization of new fcc HEAs.

The remainder of this paper is organized as follows. Section 2 introduces some basic concepts for our model of random alloys. Section 3 presents the solute strengthening theory. Section 4 discusses validation of the concepts and model using molecular simulations. Section 5 presents a reduced model based on elasticity. In Section 6, we apply the 
reduced model to predict strengthening in the $\mathrm{Ni}$ Co-Fe-Cr-Mn alloys and compare with experiments. Further discussions on implications and application of the theory are given in section 7. Several appendices contain valuable derivations or additional detail that expand on results shown in the main text.

\section{Key Model Concepts}

We consider an $N$-component random fcc HEA with concentration $c_{n}$ of the $n^{\text {th }}$ element $\left(\sum_{n=1}^{N} c_{n}=1\right)$. Typical HEAs have many elemental components at high concentrations and with local structural/compositional disorder, and so analytical theories of solute strengthening for dilute alloys with non-interacting solutes 9, 10, 11, 12, 13, 14 would not seem to apply. However, to first order, each elemental alloy component can be seen as a "solute" embedded in the average effective medium "matrix" of the surrounding material; such an effective medium approximation is well-established in different contexts such as in electronic structure theory, with the Virtual Crystal and Coherent Potential Approximations [15, 16, 17, 18, 19, 20, and in Embedded Atom Method potentials [21, 22.

We thus define here an average reference material for the HEA that has all the average properties of the true alloy: lattice constant $a$, elastic constants $\left\{C_{i j}\right\}$ including shear modulus $\mu$ and Poisson's ratio $\nu$, and stable/unstable stacking fault energies $\gamma_{\mathrm{SF}}$ and $\gamma_{\mathrm{USF}}$, all of which depend on the average alloy composition. The gliding $\{111\}(110)$ dislocations in the effective fcc matrix are like dislocations in elemental fcc metals, with a Burgers vector $b=a / \sqrt{2}$, dissociation into two Shockley partials separated by an intrinsic stacking fault, and gliding at very low Peierls stresses [22, 23]. Each individual solute then interacts with the dislocation in the average matrix, shown schematically in Fig. 1a,b, thus accounting for the solute interactions with the average chemical neighborhood. The chemicallycontrolled (non-Hall-Petch) strengthening in HEAs is then primarily the strengthening of a random solid solution with $100 \%$ solute concentration in the average matrix. This fundamental approximation of solutes in an effective matrix is the basis for our model of solute strengthening at arbitrary solute concentrations. Moreover, while the model starts from the effective medium approximation, the development below includes the additional effects due to local variations in solute chemical environment and structure around the average matrix environment.

Strengthening in the true random alloy, i.e. increased stress required to move a dislocation, arises from the totality of the interaction energies between the solutes and an individual dislocation. The effective medium approximation for the matrix allows us to consider a straight dislocation in that matrix, surrounded by solutes. The interaction energy for a solute of type $n$ located at position $\left(x_{i}, y_{j}, z_{k}\right)$ relative to the center of a straight dislocation lying along $z$ is denoted as $U^{n}\left(x_{i}, y_{j}, z_{k}\right)$ (Fig, 1, , b), and may depend on the local environment of the type $n$ solute. Contributions to this interaction energy arise from the elastic interaction of the dislocation stress field with the misfit strain tensor of the solute and from chemical-specific interactions when the solute lies in the dislocation partial cores or along the stacking fault [13, 24, 25, 26, 27]. The random distribution of solutes in the lattice gives rise to local fluctuations in solute concentrations and distortions, and the dislocation is attracted to energetically-favorable fluctuations and repelled by energetically-unfavorable fluctuations. When a straight dislocation segment of length $\zeta$ at initial position $x=0$ glides over a distance $w$, the change in the position of the dislocation relative to the solutes leads to a potential energy change of

$$
\begin{aligned}
\Delta U_{t o t}(\zeta, w)= & \sum_{i, j, k} \sum_{n} s_{i j k}^{n}\left[U^{n}\left(x_{i}-w, y_{j}, z_{k}\right)\right. \\
& \left.-U^{n}\left(x_{i}, y_{j}, z_{k}\right)\right],
\end{aligned}
$$

where $s_{i j k}^{n}=1$ if a type- $n$ solute is at position $\left(x_{i}, y_{j}, z_{k}\right)$ and 0 otherwise. The magnitude of the typical energy decrease when the dislocation segment $\zeta$ moves into a region of favorable solute fluctuations is the standard deviation of the potential energy change $\sigma_{\Delta U_{\text {tot }}}(\zeta, w)$, given by

$\sigma_{\Delta U_{\text {tot }}}(\zeta, w)=\left[\left\langle\Delta U_{t o t}^{2}(\zeta, w)\right\rangle-\left\langle\Delta U_{t o t}(\zeta, w)\right\rangle^{2}\right]^{\frac{1}{2}}$.

$\sigma_{\Delta U_{\text {tot }}}(\zeta, w)$ can be computed for a random distribution of solutes, as shown in Appendix A. In particular, since the straight dislocation segment of length $\zeta$ is invariant along the line direction $z$, an average over local variations in the interaction energies due to both local distortions and local chemical environments among the sites $z_{k}$ can be rigorously 
This is a pre-print of the following article: Varvenne, C.; Luque, A.; Curtin, W. A. Acta Mater. 2016, 118, 164-176.. The formal publication is available at http://dx.doi.org/10.1016/j.actamat.2016.07.040

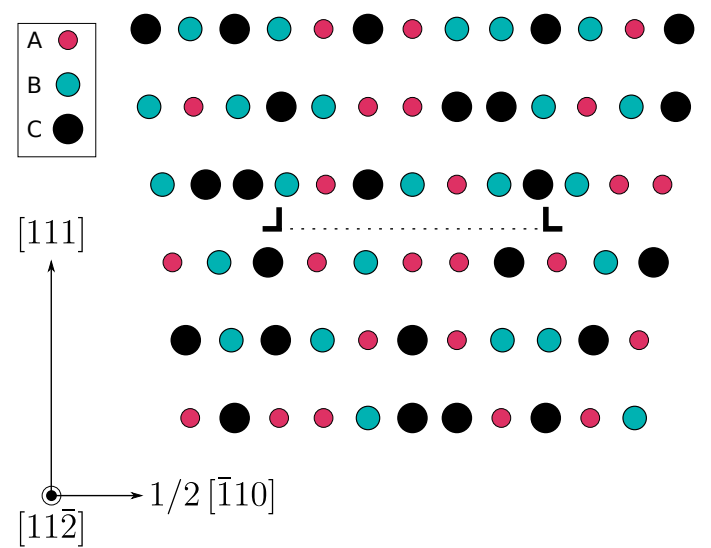

(a) real HEA

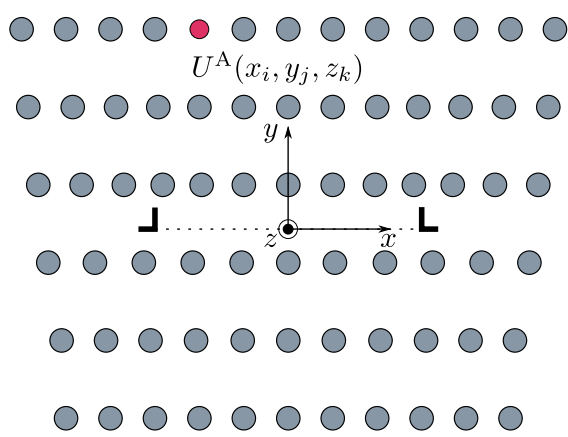

(b) effective medium

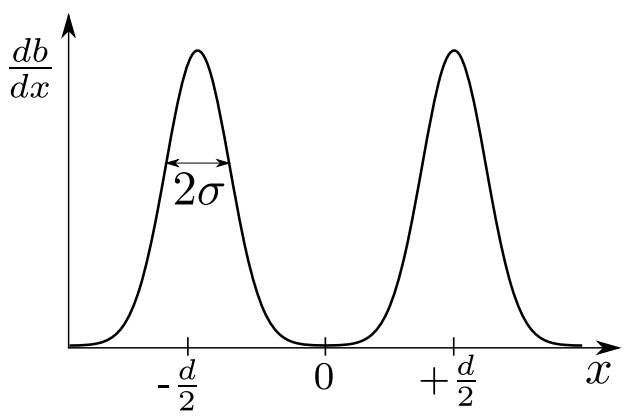

(c) Burgers vector distribution

Figure 1: Effective medium approach for dislocation/solute interactions. (a), Fully-random 3-component HEA containing a dissociated edge dislocation; (b), Effective matrix material of the same alloy, with an embedded $A$ "solute" at position $\left(x_{i}, y_{j}, z_{k}\right)$ relative to the dislocation centered at the origin, with interaction energy $U^{A}\left(x_{i}, y_{j}, z_{k}\right) ;(\mathrm{c})$, Normalized Burgers vector distribution $\frac{d b}{d x} \sim e^{-\frac{1}{2}\left(\frac{x-d / 2}{\sigma}\right)^{2}}+e^{-\frac{1}{2}\left(\frac{x+d / 2}{\sigma}\right)^{2}}$ along the glide plane of edge dislocation in the effective matrix, with $d$ the dislocation dissociation distance and $\sigma$ describing the partial spreading (see Appendix D. performed (using a mean field approximation). The final outcome of the averaging process can be expressed in the form

$\sigma_{\Delta U_{\mathrm{tot}}}(\zeta, w)=\left(\frac{\zeta}{\sqrt{3} b}\right)^{\frac{1}{2}} \Delta \tilde{E}_{p}(w)$,

with

$$
\begin{aligned}
\Delta \tilde{E}_{p}(w)= & {\left[\sum _ { \substack { i , j \\
n } } c _ { n } \left(\left(\bar{U}^{n}\left(x_{i}-w, y_{j}\right)-\bar{U}^{n}\left(x_{i}, y_{j}\right)\right)^{2}\right.\right.} \\
& \left.\left.+\sigma_{\Delta U_{i j}^{n}}^{2}\right)\right]^{\frac{1}{2}}
\end{aligned}
$$

Here, $\bar{U}^{n}\left(x_{i}, y_{j}\right)$ is the average value of $U^{n}\left(x_{i}, y_{j}, z_{k}\right)$ over the local environments along the line $z_{k}$ at in-plane position $\left(x_{i}, y_{j}\right)$, and $\sigma_{\Delta U_{i j}^{n}}$ is the associated standard deviation, which embodies the contributions due to the local environment/structural fluctuations along $z_{k} . \Delta \tilde{E}_{p}(w)$ is the key quantity for strengthening in the theory presented in the next section.

\section{Solution Strengthening Model}

The theory for solution strengthening follows the lines of a predictive model for dilute alloys [12, 27, 28. Here, we present the important steps in the derivation, highlighting key features that emerge in the context of HEAs.

\subsection{Minimum-energy dislocation configuration}

A long straight dislocation of length $L$ will reduce its total potential energy by adopting a wavy configuration, where some segments of characteristic length $\zeta_{c}$ reside in regions of favorable solute fluctuations. These segments lie at the minima in a potential energy landscape having typical minima and maxima spaced by some glide distance $w_{c}$. The pinned segments $\zeta_{c}$ are connected through additional segments $\zeta_{c}$ (Fig. 2). Dislocation bowing has an energy cost $\Delta E_{\mathrm{LT}}\left(\zeta_{c}, w_{c}\right)=\Gamma w_{c}^{2} / 2 \zeta_{c} \quad\left(w_{c} \ll \zeta_{c}\right.$, verified ex post facto), where $\Gamma$ is the dislocation line tension in the effective matrix.

To determine the length scales $\zeta_{c}$ and $w_{c}$, we first consider the total energy for arbitrary $\zeta$ and $w$, and then minimize that energy. The total energy is the 
This is a pre-print of the following article: Varvenne, C.; Luque, A.; Curtin, W. A. Acta Mater. 2016, 118, 164-176.. The formal publication is available at http://dx.doi.org/10.1016/j.actamat.2016.07.040

sum of the potential energy and elastic bowing energy, and for arbitrary $\zeta$ and $w$ is given by

$$
\begin{aligned}
\Delta E_{\mathrm{tot}}(\zeta, w) & =\Delta E_{\mathrm{LT}}(\zeta, w)-\sigma_{\Delta U_{\mathrm{tot}}}(\zeta, w)\left(\frac{L}{2 \zeta}\right) \\
& =\left[\Gamma \frac{w^{2}}{2 \zeta}-\left(\frac{\zeta}{\sqrt{3} b}\right)^{\frac{1}{2}} \Delta \tilde{E}_{p}(w)\right]\left(\frac{L}{2 \zeta}\right) .
\end{aligned}
$$

$\sigma_{\Delta U_{\text {tot }}}(\zeta, w)$ is the typical energy decrease by allowing the dislocation segment $\zeta$ to move into a region of favourable solute fluctuations, which is given by Eq. (3). $\Delta E_{\text {tot }}$ is minimized with respect to $\zeta$ and $w$ to determine the characteristic configuration $\left(\zeta_{c}, w_{c}\right)$. Minimization of Eq. (5) with respect to $\zeta$ is analytic and yields

$\zeta_{c}(w)=\left(4 \sqrt{3} \frac{\Gamma^{2} w^{4} b}{\Delta \tilde{E}_{p}^{2}(w)}\right)^{\frac{1}{3}}$.

Subsequent minimization with respect to $w$ is performed numerically, and reduces to the solution of

$\frac{\partial \Delta \tilde{E}_{p}(w)}{\partial w}=\frac{\Delta \tilde{E}_{p}(w)}{2 w}$.

Consequently, the minimized $w_{c}$ value is defined entirely by the potential energy function $\Delta \tilde{E}_{p}(w)$; this feature is important for validation using molecular simulations, as discussed below. The dislocation thus adopts a waviness at a particular amplitude $w_{c}$ determined by the potential energy function and the long dislocation line then finds the value of $\zeta_{c}$ that minimizes the total energy for a given line tension $\Gamma$

In the minimum energy configuration, each segment $\zeta_{c}$ along the wavy dislocation line sits in a local potential energy well. The depth of the well, relative to zero energy, is $-\sigma_{\Delta U_{\text {tot }}}$, and the width of the energy well ( $\min$ to $\max$ ) is $w_{c}$. This local potential energy well is approximated as a sinusoidal function: with the minimum located at $x=0$ along the glide plane, the energy of the segment of length $\zeta_{c}$ at position $x$ is $E(x)=\frac{\Delta E_{b}^{\prime}}{2}\left[1-\cos \left(\frac{\pi x}{w_{c}}\right)\right]$, with $\Delta E_{b}^{\prime}=\sqrt{2} \sigma_{\Delta U_{\text {tot }}} L / 2 \zeta_{c}$. The potential energy barrier $\Delta E_{b}^{\prime}$ is larger than $\sigma_{\Delta U_{\text {tot }}} L / 2 \zeta_{c}$ by $\sqrt{2}$, because the average barrier is the potential energy difference between the average minimum and the average maximum, not the average minimum and the zero energy level. The total energy barrier $\Delta E_{b}$ corresponds to the energy cost of moving from a favourable to an unfavorable potential energy fluctuation over a distance $w_{c}$, minus the gain in the line energy $\Delta E_{\mathrm{LT}}$,

$$
\begin{aligned}
\Delta E_{b} & =\Delta E_{b}^{\prime}-\Delta E_{\mathrm{LT}}, \\
& =1.22\left(\frac{w_{c}^{2} \Gamma \Delta \tilde{E}_{p}^{2}\left(w_{c}\right)}{b}\right)^{\frac{1}{3}} .
\end{aligned}
$$

\subsection{Thermally-activated glide}

To glide, the dislocation must overcome the barrier $\Delta E_{b}$ by thermal activation. This is facilitated by the work $-\tau b \zeta_{c} x$ of an applied resolved stress $\tau$ done on the length $\zeta_{c}$ segment as it glides a distance $x$ relative to the minimum energy position. For the sinusoidal energy well, the stress-dependent energy barrier is then accurately described [1] by the asymptotic result

$\Delta E(\tau)=\Delta E_{b}\left(1-\frac{\tau}{\tau_{y 0}}\right)^{\frac{3}{2}}$

where $\tau_{y 0}$ is the zero-temperature flow stress, i.e. the stress at which the energy barrier vanishes, and is given explicitly as

$\tau_{y 0}=\frac{\pi}{2} \frac{\Delta E_{b}}{b \zeta_{c}\left(w_{c}\right) w_{c}}=1.01\left(\frac{\Delta \tilde{E}_{p}^{4}\left(w_{c}\right)}{\Gamma b^{5} w_{c}^{5}}\right)^{\frac{1}{3}}$.

At stresses $\tau<\tau_{y 0}$, and for quasi-static loading, the plastic strain-rate $\dot{\varepsilon}$ is related to the energy barrier through a thermally-activated Arrhenius [11, 29] model: $\dot{\varepsilon}=\dot{\varepsilon}_{0} \exp (-\Delta E(\tau) / k T)$. Using the stress-dependent energy barrier of Eq. (9), we invert this relation to obtain the finite-temperature, finite strain-rate flow stress $\tau_{y}(T, \dot{\varepsilon})$ as

$\tau_{y}(T, \dot{\varepsilon})=\tau_{y 0}\left[1-\left(\frac{k T}{\Delta E_{b}} \ln \frac{\dot{\varepsilon}_{0}}{\dot{\varepsilon}}\right)^{\frac{2}{3}}\right]$.

Here, $\dot{\varepsilon}_{0}$ is a reference strain-rate that is nominally connected to the dislocation density $\rho$, Burgers vector $b$, typical dislocation slip distance $d_{s}$ and attempt frequency $\nu_{0}$ via the Orowan relationship $\dot{\varepsilon}_{0}=\rho b d_{s} \nu_{0}$. The value of $\dot{\varepsilon}_{0}$ enters only through a logarithm and so its precise value is of limited importance. We set $\dot{\varepsilon}_{0}=10^{4} \mathrm{~s}^{-1}$, consistent with previous work 12 .

The above framework dominates at low temperatures and high stresses. At higher temperatures/lower stresses, the dislocation can explore 
This is a pre-print of the following article: Varvenne, C.; Luque, A.; Curtin, W. A. Acta Mater. 2016, 118, 164-176.. The formal publication is available at http://dx.doi.org/10.1016/j.actamat.2016.07.040

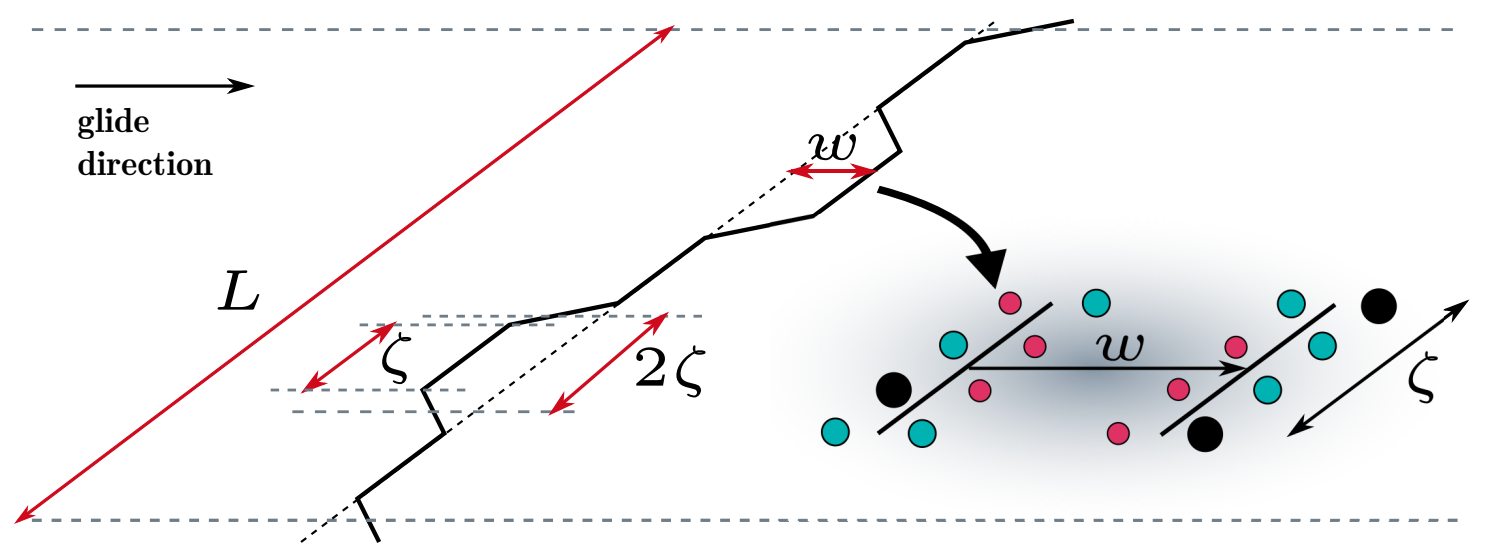

Figure 2: Schematic of the low-energy wavy configuration of the dislocation as it moves through the random field of solutes. The configuration is characterized by segments of lateral length $2 \zeta$ of amplitude $w$ along the length of the long dislocation. The key quantity is the change in energy of a straight segment of length $\zeta$ as it glides a distance $w$ through the random solute field. The total dislocation energy is minimized with respect to both $\zeta$ and $w$ to obtain the controlling characteristic lengths $\zeta_{c}$ and $w_{c}$.

additional longer-wavelength configurations superimposed on the underlying scale of $\zeta_{c}, w_{c}$, as first proposed by Labusch [30] and recently formalized and quantified by Leyson et al. 31. However, the thermally-activated finite-temperature strength still scales with the energy barrier $\Delta E_{b}$, but strength given by 27 .

$\tau_{y}(T, \dot{\varepsilon})=\tau_{y 0} \exp \left(-\frac{1}{0.51} \frac{k T}{\Delta E_{b}} \ln \frac{\dot{\varepsilon}_{0}}{\dot{\varepsilon}}\right)$.

The low-T/high-stress and high-T/low-stress results above are essentially equal over the range $0.3 \leq \tau_{y} / \tau_{y 0} \leq 0.6$.

\subsection{Strengthening in HEAs}

By starting from an effective medium matrix, the theory first averages out the effects of all the solutes and then reintroduces the effects of solute fluctuations in attracting and repelling a dislocation. The theory considers all possible scales of fluctuation $(\zeta, w)$ in the random alloy, over scales where line tension is suitable for evaluating the elastic energy 24] of the non-straight dislocation configurations. The theory thus naturally identifies that there are mesoscale collective concentration/structural fluctuations on the scale of $\left(\zeta_{c}, w_{c}\right)$ that create the dominant energy barrier controlling the yield stress [32] in the random alloy. The dislocation does not respond to smaller-scale fluctuations because they are energetically costly, even though such fluctuations certainly exist.

The critical quantities, energy barrier $\Delta E_{b}$ and zero-temperature yield stress $\tau_{y 0}$, depend on the key energy $\Delta \tilde{E}_{p}\left(w_{c}\right)$ which is computed here for random fcc alloys of any composition and number of components (see Eq. (4)). The combination of Eqs. (4) and (8) - (11) then constitutes the general theory for strengthening in HEAs, and is the first main result of this work.

The theory does not consider atomic fluctuations at the scale of $b<\zeta_{c}, w_{c}$ because the line tension concept would not apply [24]. But, such fluctuations could occur - they are just not calculable at the present time. In fact, $\AA$-scale fluctuations in the dislocation position are indeed observed in simulations, and are likely due to additional terms in the potential energy that are not captured by either $\bar{U}^{n}\left(x_{i}, y_{j}\right)$ or its fluctuations $\sigma_{\Delta U_{i j}^{n}}$. However, such small-scale fluctuations occur within the larger mesoscale energy landscape, not in place of it. While not calculable, such fluctuations could generate small additional energy barriers that would contribute to strengthening at zero temperature, but would be expected to be easily overcome at finite temperature. 


\section{Molecular Statics Validation}

The model presented here is based on several approximations, and so some independent validation is valuable to assess the accuracy of the model. To this end, we perform a comparison between key aspects of the model and direct molecular statics (MS) simulations of the flow stress. We study Fe-Ni-Cr fcc alloys, described using the Embedded Atom Method (EAM) potentials developed in Ref. [33], as a convenient well-defined multicomponent system.

\subsection{Flow-stress of a straight dislocation segment}

Direct molecular simulations cannot capture the evolution of wavy configurations of long dislocations $\left(L \gg \zeta_{c}\right)$ with energy barriers on the order of $\sim 0.7-1.1 \mathrm{eV}$ at experimental strain-rates. However, the stress required to move a single straight dislocation segment of length $\zeta \leq \zeta_{c}$ out of its initial local energy minimum at $T=0 K$ can be simulated and can be calculated using the theory. Theoretically, at length $\zeta \leq \zeta_{c}$ the dislocation remains straight and moves through the random potential energy landscape with no bowing. The potential energy lanscape has maxima and minima spaced on the scale of $w_{c}$. We can thus introduce a straight dislocation into the material, let it relax to its local energy minimum, and then measure the stress required to push the dislocation out of the local minima, over the local maximum.

The theoretical prediction for the stress required to move an initial straight dislocation of length $\zeta=\zeta_{c}$ out of its local potential energy well is derived by considering only the potential energy of interaction with solutes, i.e. there is no bowing and no line-tension energy cost associated with moving of the straight dislocation. Therefore, an average dislocation straight segment starts in an average local energy minimum of depth $\sigma_{\Delta U_{\text {tot }}}$ (absolute value), relative to the zero energy level. The dislocation must escape over a barrier that is $\sqrt{2}$ larger and located at a distance $w_{c}$ from the minimum. Again, an applied stress provides the energy (work) necessary to escape over the barrier at zero temperature. Thus, similar to Eqs. (9) and (10), the predicted average zero-temperature flow stress $\tau_{y 0}^{\mathrm{SS}}$ for one segment of length $\zeta=\zeta_{c}$ moving through a random landscape varying on length scale $w_{c}$ is

$$
\begin{aligned}
\tau_{y 0}^{\mathrm{SS}} & =\frac{\pi}{2} \frac{1}{b \zeta_{c} w_{c}} \sqrt{2}\left(\frac{\zeta_{c}}{\sqrt{3} b}\right)^{\frac{1}{2}} \Delta \tilde{E}_{p}\left(w_{c}\right), \\
& =\frac{1.69}{b^{\frac{3}{2}} \zeta_{c}^{\frac{1}{2}} w_{c}} \Delta \tilde{E}_{p}\left(w_{c}\right) .
\end{aligned}
$$

For the assumed local sinusoidal potential energy landscape, the theory also predicts the average dislocation position $x$ versus stress $\tau$ as the stress pushes the dislocation up the underlying energy barrier, $x=\left(w_{c} / \pi\right) \arcsin \left(\tau / \tau_{y 0}^{\mathrm{SS}}\right)$. The dislocation escapes thus when $x=w_{c} / 2$ at $\tau_{y 0}^{\text {SS }}$.

The above pertains to the average local minimum. Simulations will sample the statistical distribution of local minimum, both weaker and stronger binding, with variations in the local width of the potential energy landscape (e.g. a specific $w_{c}$ for each case). Thus, for one given alloy composition, multiple simulations are needed to obtain the average behavior for comparison with the theory. We now turn to the simulations on the model alloys.

\section{2. $M S$ vs. Theory: $\mathrm{Fe}-\mathrm{Ni}-\mathrm{Cr}$ model alloys}

We focus on $\mathrm{Fe}_{(1-x) / 2} \mathrm{Ni}_{(1-x) / 2} \mathrm{Cr}_{x}$ fcc alloys, with different $x$ values, because our studies reveal that the $\mathrm{Cr}$ content controls many property variations. Within EAM description of alloys energetics, a rigourous analytical configurational averaging procedure 22] leads to an average-atom EAM potential that represents the effective medium matrix for the multicomponent alloy at any desired composition. The resulting average-atom potential captures many average properties of the random alloys, including defect properties, with all fluctuations averaged out analytically. It can be used to perform usual molecular simulations, and thus allows to measure the Peierls stresses and all model inputs for the different $\mathrm{Fe}-\mathrm{Ni}-\mathrm{Cr}$ random alloys.

The Peierls stresses of an $\{111\}(100)$ edge dislocation in the effective matrix alloys are measured using standard methods, and are 10, 3.5, 1.5, 3 and $3 \mathrm{MPa}$ for $x=10,20,33,40$ and $50 \%$, respectively. These values are very small, as expected for singleelement fcc materials. This result alone shows that is it precisely the chemical and structural fluctuations that cause the high strength in HEA materials. Figs. 3a,b show $\mu, \gamma_{\mathrm{SF}}$, and $\gamma_{\mathrm{USF}}$ versus $x$ for these alloys, demonstrating significant variations in key dislocation-related material parameters versus alloy composition. 
This is a pre-print of the following article: Varvenne, C.; Luque, A.; Curtin, W. A. Acta Mater. 2016, 118, 164-176.. The formal publication is available at http://dx.doi.org/10.1016/j.actamat.2016.07.040

The inputs to the theory are the average interaction energies $\bar{U}^{n}\left(x_{i}, y_{j}\right)$ between solutes $n=\mathrm{Fe}$, $\mathrm{Ni}, \mathrm{Cr}$ and a straight edge dislocation in the effective matrix. These are computed directly as the energy change between a simulation cell having a substitutional single solute atom at the desired position near the dislocation into the effective medium matrix, and a simulation cell with the same solute but far from the dislocation, thus non-interacting with it 22. The results are shown in Fig. 4 for $x=10 \%$ and $x=50 \%$ and for solute positions in and around one of the two dissociated partials (behavior around the other partial is the same). The solute interactions also vary significantly with composition: at $x=10 \%$, interactions with the partial core are nearly zero for Fe but very strong for Cr, whereas moderate interactions exist for all solutes at $x=50 \%$. The fluctuations in the interaction energies due to local environments cannot be computed in the average matrix, and are thus neglected, i.e. $\sigma_{\Delta U_{i j}^{n}}^{2}=0$ here. The full theory then determines $\left(\zeta_{c}, w_{c}\right)$, both of which also vary strongly with composition $x$ as shown in Fig. 3. All these wide variations in "matrix" properties, "solute" properties, and in characteristic mesoscale lengths across these model alloys precludes any a priori conclusion on the flow strengths of these different EAM alloys. The theory, however, automatically considers all of these factors versus composition and has no adjustable parameters.

MS simulations of the flow stresses are performed on twenty different random realizations, per alloy composition, of dislocation segments of length $\sim \zeta_{c}$ moving under the action of an increasing shear stress (see Appendix C for details). It is important to note that, once it escapes from its local minimum, a straight dislocation segment will always be halted at a next-stronger-fluctuation arising statistically further along the glide plane. The stress vs. position is thus always monotonically increasing. The "strength" measured in the MS simulations therefore depends on the glide distance chosen. The theory for escape from the average barrier predicts that the average strength is attained at a glide distance of $w_{c} / 2$, and so comparisons of averages are made at this glide distance. The successive pinning in the MS of a single straight segment does not arise in a real material because a very long dislocation line length $L \gg \zeta_{c}$ will bowout around any rare stronger pinning regions and so the strength will be controlled by the average pin-

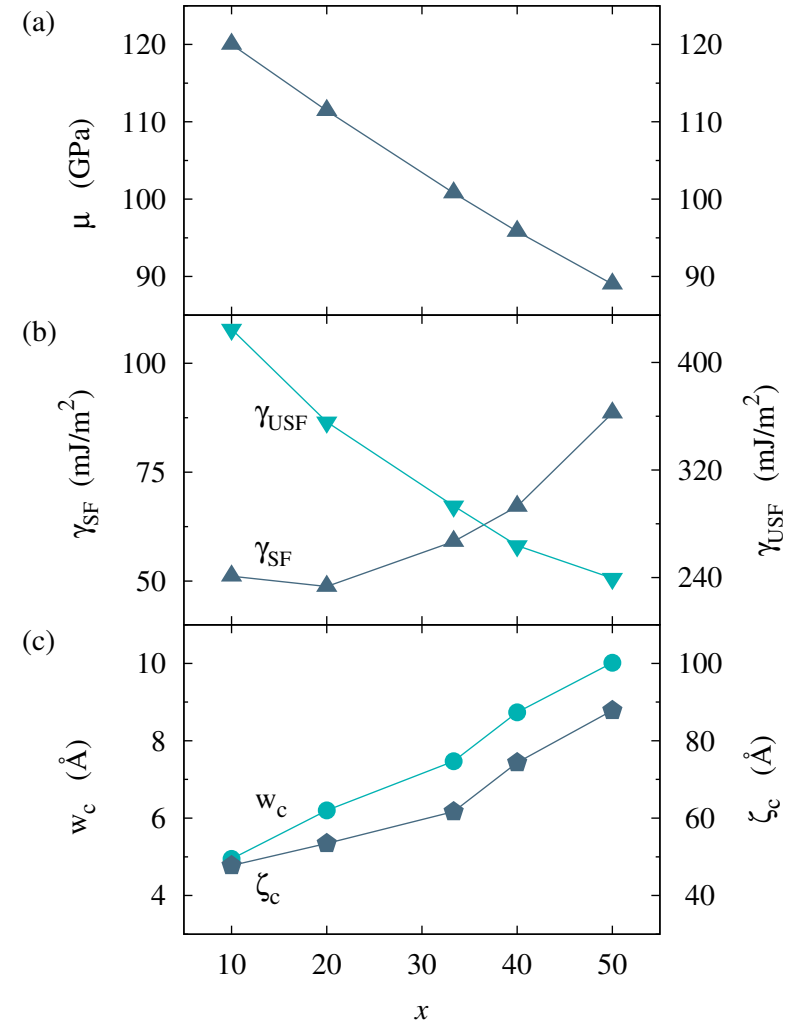

Figure 3: Effective matrix properties for different $\mathrm{Fe}_{(1-x) / 2} \mathrm{Ni}_{(1-x) / 2} \mathrm{Cr}_{x}$ EAM alloys: (a), $\mu_{\text {Voigt }}$, and (b), $\gamma_{\mathrm{SF}}$ and $\gamma_{\mathrm{USF}}$. (c), Characteristic lengths $\left(w_{c}, \zeta_{c}\right)$ for the same alloys, as predicted by the full strengthening theory.

ning environment predicted by the theory. With this point in mind, Fig. 5 a shows the applied shear stress as a function of the measured average glide position of the dislocation $x$, relative to its initial position in the energy minimum $x=0$, averaged over twenty samples at the two extreme compositions of $\mathrm{Fe}_{45} \mathrm{Ni}_{45} \mathrm{Cr}_{10}$ and $\mathrm{Fe}_{25} \mathrm{Ni}_{25} \mathrm{Cr}_{50}$ (see Appendix C for full data). Also shown are the theoretical predictions for stress versus glide distance, with the dislocation predicted to unpin at $x=w_{c} / 2$ at stress $\tau_{y 0}^{S S}$. The averaged simulations agree well with the theory for glide distances up to $w_{c} / 2$ and then show the slow steady increase for glide beyond $w_{c} / 2$ as expected due to features of the MS simulation discussed above. The theory thus captures the relevant internal length scale $w_{c}$ controlling the dislocation strength as a function of alloy composition, which is an important test of the theory. Moreover, Fig. $5 \mathrm{~b}$ shows the predicted and simulated average flow stress at $x=w_{c} / 2$. Excellent agreement is 
This is a pre-print of the following article: Varvenne, C.; Luque, A.; Curtin, W. A. Acta Mater. 2016, 118, 164-176.. The formal publication is available at http://dx.doi.org/10.1016/j.actamat.2016.07.040

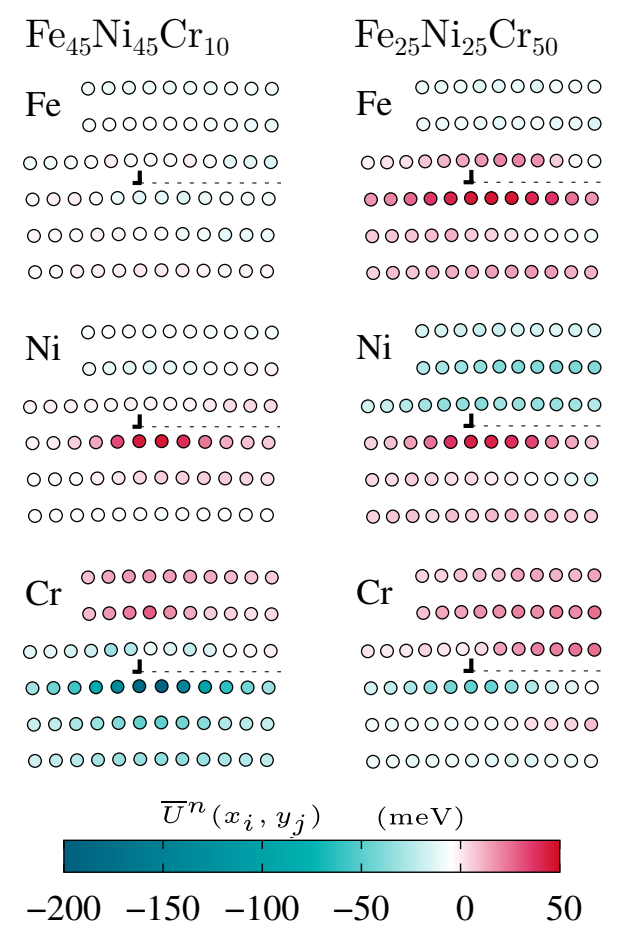

Figure 4: Interaction energies between one partial dislocation in the effective alloy matrix and each of the solutes $\mathrm{Fe}$, $\mathrm{Ni}$ and $\mathrm{Cr}$, in $\mathrm{Fe}_{(1-x) / 2} \mathrm{Ni}_{(1-x) / 2} \mathrm{Cr}_{x}$ EAM alloys and for the limiting compositions $x=10$ (right) and $50 \%$ (left).

found between theory and simulations across the five $\mathrm{Fe}-\mathrm{Ni}-\mathrm{Cr}$ compositions possessing a range of underlying material properties. The low-Cr alloys are much stronger than the high-Cr alloys, and the $w_{c}$ much smaller, because the $\mathrm{Cr} /$ dislocation interaction energies in the dislocation partial cores are much larger in the low-Cr alloys (see Fig. 4). It is important to keep in mind that in the absence of the theory there would be zero ability to predict either this magnitude of strengths (recall the Peierls stresses are negligible) nor the detailed trends with alloy composition.

If we wished to be more conservative in making comparisons, we could measure the "strength" in the simulations at $x=3 w_{c} / 4$, a point at which $>18$ of the 20 samples have experienced some very large jump forward in dislocation position, indicative of unpinning from a local energy well. The "strengths" measured in simulations using this larger distance are 15-25\% larger than the theory, still in good agreement with, and following the trends of, the parameter-free theory.

The overall qualitative and quantitative success of the parameter-free theory, in terms of not only strength but also the distribution in strength and the length scales controlling the strength versus alloy composition, for a range of model materials having considerable variations in many material properties, is the second main result of this paper.

\section{Reduced Model: Elastic Interactions}

The full general model does not provide significant insights into the role of the average matrix or solute properties, nor dislocation structure, and full solute/dislocation interaction energies $\bar{U}^{n}\left(x_{i}, y_{j}\right)$ may not be easily computable or measurable in real materials. We thus now reduce the theory to a widely-usable form by considering only the main elasticity contribution $U_{\mathrm{el}}^{n}\left(x_{i}, y_{j}, z_{k}\right)=$ $-p\left(x_{i}, y_{j}\right) \Delta V_{n}\left(x_{i}, y_{j}, z_{k}\right)$ to the solute/dislocation interaction energy, which is due to the interaction between the pressure field $p\left(x_{i}, y_{j}\right)$ of the dislocation at the solute site and the solute $n$ misfit volume at this site. This contribution is common to all fcc HEAs and, due to symmetry, local deviatoric misfit strains must average to zero and so would only appear in the secondary local fluctuation term. We express the dislocation pressure field as $p\left(x_{i}, y_{j}\right)=-\frac{\mu}{3 \pi} \frac{(1+\nu)}{(1-\nu)} f\left(x_{i}, y_{j}\right)$ where $f\left(x_{i}, y_{j}\right)$ is the dimensionless anisotropic pressure field generated by the distribution of normalized Burgers vector along the glide plane (see Fig. 1 1 ) with $\mu$ and $\nu$ the isotropic elastic constants introduced for scaling purposes. Inserting these into Eq. (1), the key energy in the theory becomes

$$
\begin{aligned}
\Delta \tilde{E}_{p}(w)= & \frac{\mu}{3 \pi} \frac{(1+\nu)}{(1-\nu)}\left[\sum_{i, j} \Delta f_{i j}^{2}(w)\right]^{\frac{1}{2}} \\
& \times\left(\sum_{n} c_{n}\left(\Delta \bar{V}_{n}^{2}+\sigma_{\Delta V_{n}}^{2}\right)\right]^{\frac{1}{2}},
\end{aligned}
$$

where $\Delta f_{i j}(w)=f\left(x_{i}-w, y_{j}\right)-f\left(x_{i}, y_{j}\right)$. The quantity $\sum_{n} c_{n}\left(\Delta \bar{V}_{n}^{2}+\sigma_{\Delta V_{n}}^{2}\right)$ is the key misfit volume quantity that is closely related to the socalled misfit parameter $\delta$, (see Appendix B), with $\Delta \bar{V}_{n}$ the average misfit volume of solute $n$ and $\sigma_{\Delta V_{n}}$ its standard deviation due to local fluctuations. Minimization of the total energy with respect to $w$ to obtain $w_{c}$ is then determined only by $\Delta f_{i j}(w)$, which depends only on the dislocation core structure, independent of the solute properties. 
This is a pre-print of the following article: Varvenne, C.; Luque, A.; Curtin, W. A. Acta Mater. 2016, 118, 164-176.. The formal publication is available at http://dx.doi.org/10.1016/j.actamat.2016.07.040

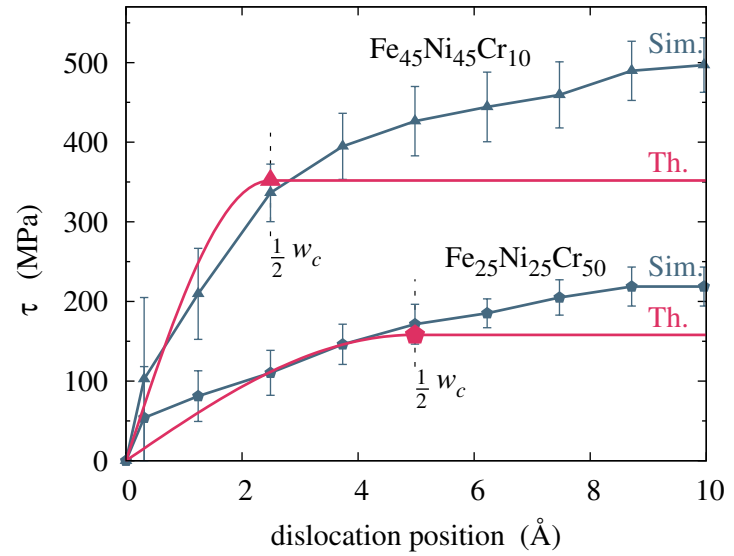

(a)

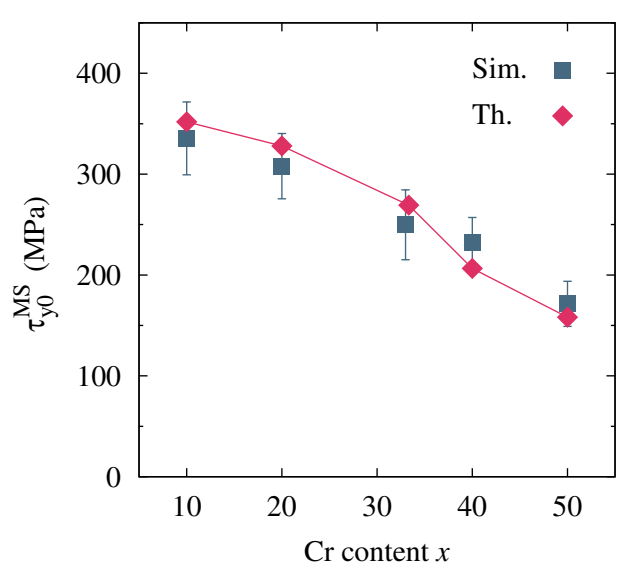

(b)

Figure 5: Validation of the theory against Molecular Statics simulations for $\mathrm{Fe}_{(1-x) / 2} \mathrm{Ni}_{(1-x) / 2} \mathrm{Cr}_{x} \mathrm{EAM}_{\mathrm{Alloys}}$ (a), Average shear stress in MS simulations vs. average dislocation position, for $x=0.1$ and $x=0.5$ alloys. Red symbols indicate predictions of the theory at the average depinning point $w_{c} / 2$. (b), Average zero-temperature flow stresses for the 5 alloys as measured in simulations (grey squares) and as predicted using Eq. 13. (red diamonds). Error bars show the confidence intervals of the mean strength obtained from simulations on 20 samples per alloy.

For a given core structure and thus given dimensionless pressure field $f\left(x_{i}, y_{j}\right)$, minimization of the total energy yields the $\left(\zeta_{c}, w_{c}\right)$, and then the theory predicts $\tau_{y 0}$ and $\Delta E_{b}$ as

$$
\begin{aligned}
\tau_{y 0}= & 0.051 \alpha^{-\frac{1}{3}} \mu\left(\frac{1+\nu}{1-\nu}\right)^{\frac{4}{3}} f_{1}\left(w_{c}\right) \\
& \times\left[\frac{\sum_{n} c_{n}\left(\Delta \bar{V}_{n}^{2}+\sigma_{\Delta V_{n}}^{2}\right)}{b^{6}}\right]^{\frac{2}{3}}, \\
\Delta E_{b}= & 0.274 \alpha^{\frac{1}{3}} \mu b^{3}\left(\frac{1+\nu}{1-\nu}\right)^{\frac{2}{3}} f_{2}\left(w_{c}\right) \\
& \times\left[\frac{\sum_{n} c_{n}\left(\Delta \bar{V}_{n}^{2}+\sigma_{\Delta V_{n}}^{2}\right)}{b^{6}}\right]^{\frac{1}{3}} .
\end{aligned}
$$

where we introduced the minimized core coefficients $f_{1}\left(w_{c}\right)=\left[\left(\frac{b}{w_{c}}\right)^{5 / 2} \sum_{i, j} \Delta f_{i j}^{2}\left(w_{c}\right)\right]^{2 / 3}$ and $f_{2}\left(w_{c}\right)=\left[\left(\frac{w_{c}}{b}\right)^{2} \sum_{i, j} \Delta f_{i j}^{2}\left(w_{c}\right)\right]^{1 / 3}$, and where the line tension is expressed here as $\Gamma=\alpha \mu b^{2}$, with $\alpha$ a dimensionless number.

To actually execute the minimization, we use isotropic elasticity to predict both dislocation core structure and associated pressure field; extension to anisotropic elasticity will be deferred to future work. We first parameterize the continuous distribution of Burgers vectors along the glide plane of the dissociated dislocation as two Gaussian peaks each of standard deviation $\sigma=1.5 b$ separated by a stacking fault of width $d=\frac{\mu b^{2}}{\gamma_{\mathrm{SF}}} \frac{(2+\nu)}{24 \pi(1-\nu)}$ [34] (see Fig. 1p). This form is consistent with atomistic studies of fcc dislocations (see Appendix D). The dimensionless pressure field $f\left(x_{i}, y_{j}\right)$ can then be computed, and the minimized core coefficients in Eqs. 15 and (16) can be obtained as a function of partial spacing $d$, as shown in Fig. 6. Interestingly, for moderate separations $d / b>10$, the minimized "core" parameters are essentially independent of $d$, with the core coefficients for $\tau_{y 0}$ and $\Delta E_{b}$ being $f_{1}\left(w_{c}\right)=0.35$ and $f_{2}\left(w_{c}\right)=5.70$. A small discontinuity is seen at small $d$ values, due to a change in $w_{c}$, which can only take on integer multiples of $b / 2$. For typical HEA elastic moduli and with estimates of stacking fault energies in HEAs 35] being below $100 \mathrm{~mJ} / \mathrm{m}^{2}$, and typically much smaller, we conclude that the stacking fault energy value is not important for the low-temperature strength in HEA materials. As an aside, we note that minimization reveals the existence of a second minimum energy configuration, with low $\tau_{y 0}$ and high $\Delta E_{b}$; this provides a "plateau" stress relevant only at very high temperatures above temperatures of interest here, and so this is not discussed further. 


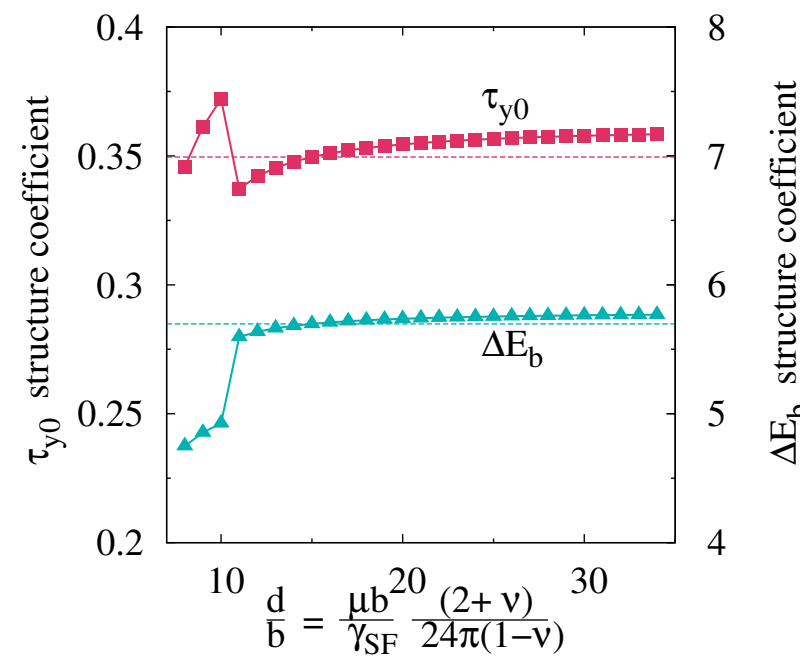

Figure 6: Dependence of strength and energy barrier on dislocation dissociation distance $d$ (related to $\gamma_{\mathrm{SF}}$ as indicated). Minimized dislocation structural coefficient for the energy barrier $\Delta E_{b}$ vs. $d$ appearing in Eq. 16 (red squares) and for the zero-temperature strength $\tau_{y 0}$, vs. $d$ appearing in Eq. 15 (blue triangles). These coefficients are nearly independent of $\gamma_{\mathrm{SF}}$. The discontinuity at small $d$ is due to a small change in the $w_{c}$ value emerging from the minimization. Dashed lines indicate values used for predictions of HEA strengths.

The reduced theory based on elasticity and misfit volumes, leading to Eqs. (15) and (16), with the computed core coefficients independent of core partial spacing, is not only simplified but is fully analytic. Predictions require only elastic moduli, lattice constants, and accurate solute misfit volumes versus alloy composition; this is the third main result of this work.

\section{Comparison to Experiments}

We now apply the analytic theory to predict the strengths of equiatomic fcc alloys in the Ni-Co-FeCr-Mn family. The uniaxial tensile yield strengths versus temperature, strain-rate, and grain size $d_{g}$ in polycrystalline materials have been measured 4, 5, 8, and show two contributions: a grain-sizedependent Hall-Petch (H-P) contribution $\sigma_{\mathrm{H}-\mathrm{P}}\left(d_{g}\right)$ and a chemical/alloying effect $\sigma_{\text {alloy }}$. These two contributions to strength have distinctly different physical origins; the present theory accounts for the chemical/alloying contribution $\sigma_{\text {alloy }}$. We therefore compare our theoretical predictions of $\sigma_{\text {alloy }}$ to experiments on those alloys for which the measured
$\mathrm{H}-\mathrm{P}$ contribution $\sigma_{\mathrm{H}-\mathrm{P}}\left(d_{g}\right)$ can be subtracted from the total experimental strength; this is standard metallurgical practice.

Polycrystalline elastic constants $(\mu, \nu)$ versus temperature (for $\mathrm{NiCoFeCr}$ and $\mathrm{NiCoFeCrMn}$ ) and at room temperature (for $\mathrm{NiCo}, \mathrm{NiCoFe}$, and $\mathrm{NiC}$ oCr) [4, 8, and the average Burgers vectors were experimentally measured (see Table 1). Average misfit volumes $\Delta \bar{V}_{n}$ of the Fe, Co and Cr solutes are calculated from the measured average atomic volumes of $\mathrm{Ni}_{1-c_{n}} \mathrm{X}_{c_{n}}$ binary fcc solid solutions with $\mathrm{X}=\mathrm{Fe}, \mathrm{Co}$ and $\mathrm{Cr}$ 21, 38, 39. The measurements show a linear variation with $c_{n}$ in the binaries. Applying Vegard's law to the average atomic volume of each solid solution, i.e. $\bar{V}=\left(1-c_{n}\right) V_{\mathrm{Ni}}+c_{n} V_{n}$, with

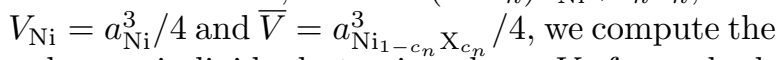
unknown individual atomic volume $V_{n}$ for each element $n$, and get $V_{n}=10.94,11.12,12.09,12.27 \AA^{3}$ for $\mathrm{Ni}, \mathrm{Co}, \mathrm{Fe}$ and $\mathrm{Cr}$, respectively. $V_{\mathrm{Mn}}=12.60 \AA^{3}$ is then obtained from the measured atomic volumes of equiatomic NiCoFeCrMn, NiCoFeMn, NiCoCrMn, NiFeMn and NiCoMn alloys, using the previously-determined $V_{n}$ for the other elements. The misfit volume of an element $n$ in a given HEA is computed as $\Delta \bar{V}_{n}=V_{n}-\bar{V}$, with $\bar{V}=\sum_{n} c_{n} V_{n}$ (the sum rule $\sum_{n} c_{n} \Delta \bar{V}_{n}=0$ is followed by construction). The predicted average Burgers vector of each HEA is computed through $b=(4 \bar{V})^{1 / 3} / \sqrt{2}$. While magnetism in the binary alloys may affect the deduced atomic volumes, the values we obtain for this set of $\left\{V_{n}\right\}$ give very good predictions for the measured overall Burgers vector $b$ of all the various HEAs, as shown in Table 1. We note that misfit volumes in HEAs are often computed using textbook "atomic" radii for the elements [40], independent of chemical, magnetic, or crystallographic structural details, and so the values here are a significant improvement over the standard estimates. Fluctuations in misfit volumes and local deviatoric misfit strains are not available experimentally and so are neglected in making predictions. The line tension parameter $\alpha=0.123$ is obtained from atomistically-measured edge dislocation line tension in the EAM FeNiCr effective matrix and is close to the coefficient for elemental $\mathrm{Al}$ 41. The analytic theory of Eqs. (15) and (16) then predicts the critical resolved shear strength versus temperature and strain-rate for any alloy composition. The corresponding uniaxial yield stress $\sigma_{\text {alloy }}$ for an equiaxed fcc polycrystal is obtained by multiplying by the Taylor factor of 3.06 .

Figs. $7 \mathrm{a}, \mathrm{b}$ show the predicted and measured al- 
This is a pre-print of the following article: Varvenne, C.; Luque, A.; Curtin, W. A. Acta Mater. 2016, 118, 164-176.. The formal publication is available at http://dx.doi.org/10.1016/j.actamat.2016.07.040

Table 1: Experimental data on the fcc Ni-Co-Fe-Cr-Mn family high entropy alloys. Burgers vector $b$ and isotropic elastic constants [4] $(\mu, \nu)$ measured at room temperature, and elastic constants versus temperature for equiatomic fcc $\mathrm{NiCoFeCr}$ [4] and $\mathrm{NiCoFeCrMn} \mathrm{36} \mathrm{37} \mathrm{alloys.} \mathrm{Burgers} \mathrm{vectors} \mathrm{obtained} \mathrm{with} \mathrm{the} \mathrm{procedure} \mathrm{described} \mathrm{in} \mathrm{the} \mathrm{text} \mathrm{are} \mathrm{given} \mathrm{for} \mathrm{comparison.}$ Estimates of the intrinsic stacking fault energies $\gamma_{\mathrm{SF}}$ are obtained by a combination of experiments and DFT calculations [35]. The corresponding dissociation distances for edge dislocation $\frac{d}{b}=\frac{\mu b}{\gamma_{\mathrm{SF}}} \frac{(2+\nu)}{24 \pi(1-\nu)}$ are given between parenthesis.

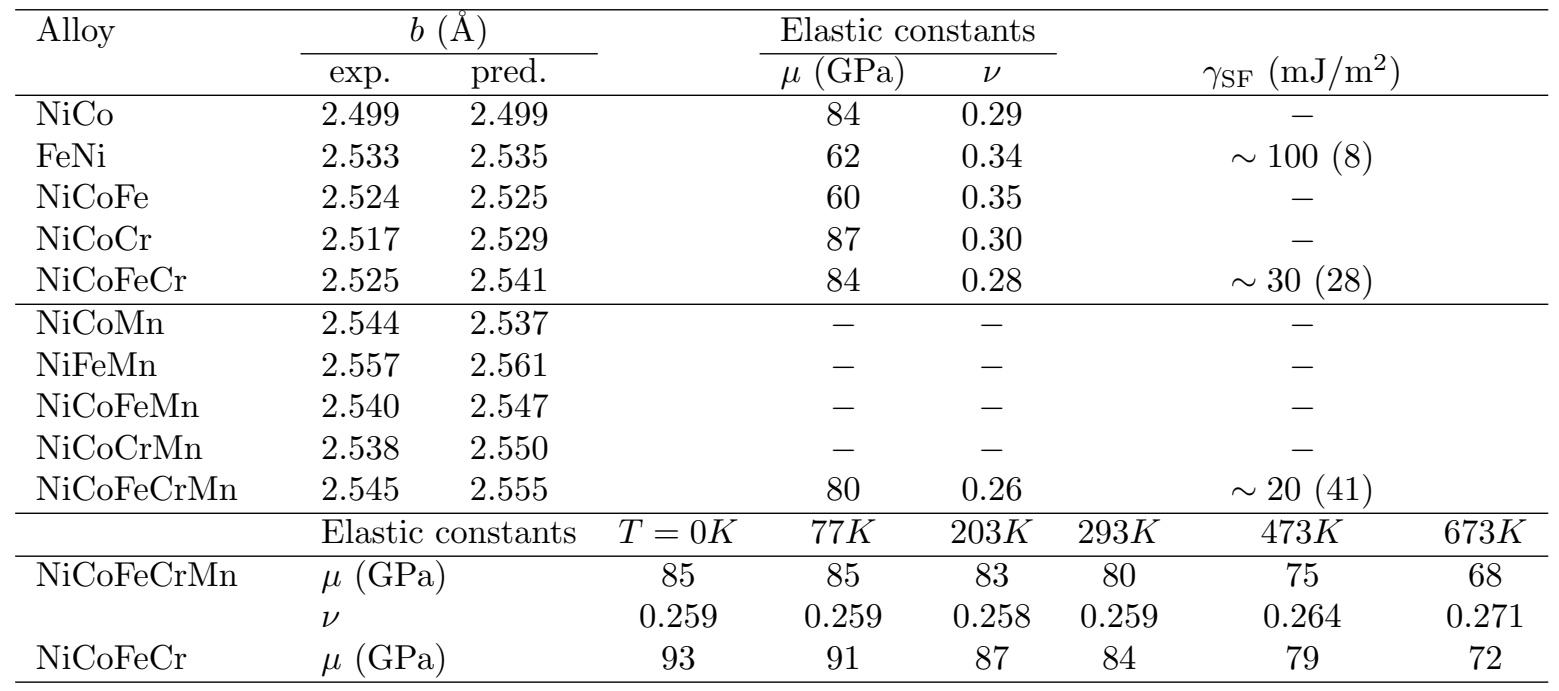

loy contributions $\sigma_{\text {alloy }}$ to the yield stress for the $\mathrm{NiCoFeCr}$ and NiCoFeCrMn alloys over the complete temperature range studied at the experimental strain-rate $\dot{\varepsilon}=10^{-3} \mathrm{~s}^{-1}$. The predictions are very good, with no fitting parameters. The experimental strengths of $\mathrm{NiCoFeCr}$ and $\mathrm{NiCoFeCrMn}$ are nearly identical, as predicted. The NiCoFeCrMn alloy has a larger misfit contribution but a lower $\mu$. In the theory, these two factors nearly cancel for the zero-temperature strength and give a slightly lower energy barrier, thus rationalizing the similarity in measured strengths. The predictions at the lowest temperature $(T=77 K)$ are below the experiments. As mentioned earlier, low$\mathrm{T}$ behavior could be affected by the neglect of $\AA$ scale fluctuations. Our predictions could also be refined using specific solute-core interactions, and the interaction-fluctuation contribution $\sigma_{\Delta U_{i j}^{n}}$, but these quantities are not available to date. Figs. $7 \mathrm{a}, \mathrm{b}$ also show predictions using a line tension parameter $\alpha=0.06125$, demonstrating that the strength predictions are nearly independent of line tension except at $T=77 \mathrm{~K}$. Low-T predictions would thus require a more precise determination of the line tension coefficient $\alpha$.

For four other alloys (NiCo, NiFe, NiCoFe, NiCoCr), the Hall-Petch strengthening was only measured at $T=293 \mathrm{~K}$ by Vickers Hardness $(H V)$ [ $]$.
We use a linear correlation between the Hall-Petch trend for $H V$ and for the yield stress to obtain $\sigma_{\text {alloy }}$ for these materials. Fig. 7k shows the parameterfree prediction of $\sigma_{\text {alloy }}$ versus the measured values for all six alloys at $T=293 K$. The overall agreement is again very good, with accuracy levels similar to those achieved in simpler dilute binary alloys [14, 27, 28. The theory also preserves the observed ordering of strength versus composition, with the $\mathrm{NiCoCr}$ alloy being the strongest of all the alloys tested. The quantitative success of the analytic model with no fitting parameters, over a wide temperature range for two HEAs and at $T=293 \mathrm{~K}$ for six HEAs, is strong evidence of the predictive capability of the model. This is the fourth main result of this work.

\section{Implications and design guidelines for HEAs}

The theory answers many open questions about strengthening in HEAs: (i) strength does not directly depend on the number of components $N$, and is not maximized by the equi-atomic composition, (ii) the strongest and most temperatureinsensitive materials are achieved by maximizing the concentration-weighted mean-square misfit volume quantity and/or increasing the shear modulus, 
This is a pre-print of the following article: Varvenne, C.; Luque, A.; Curtin, W. A. Acta Mater. 2016, 118, 164-176.. The formal publication is available at http://dx.doi.org/10.1016/j.actamat.2016.07.040

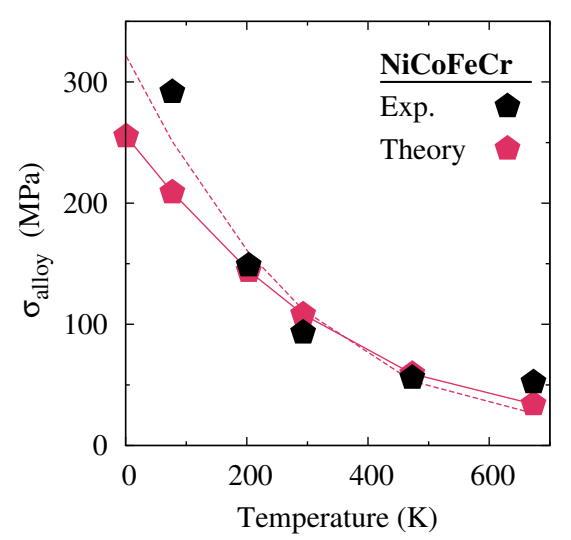

(a)

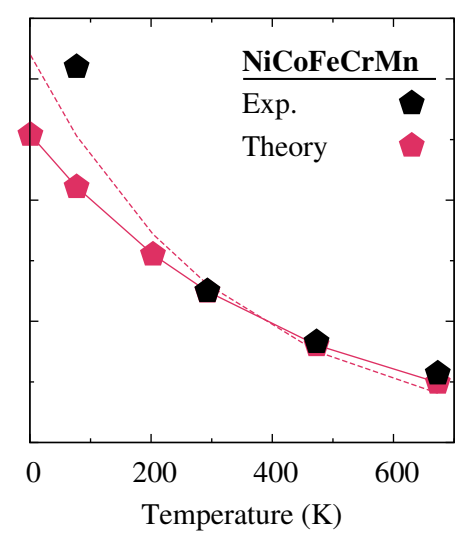

(b)

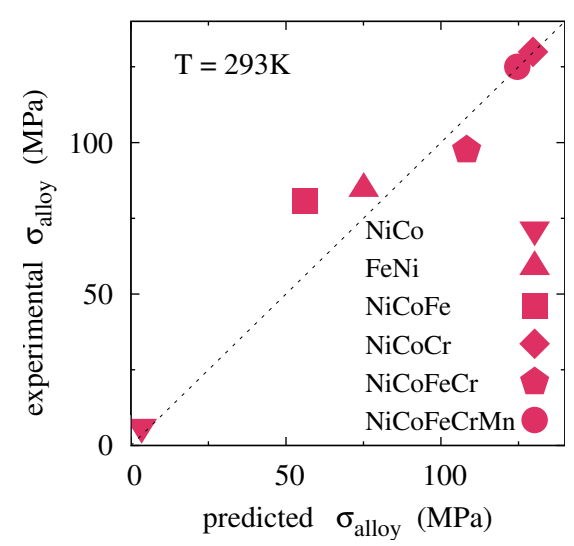

(c)

Figure 7: Quantitative comparison between experiment and theory. (a) and (b), Yield stress vs. temperature for the $\mathrm{NiCoFeCr}$ and $\mathrm{NiCoFeCrMn}$ equiatomic alloys [4, 8, as measured (black symbols) after subtraction of the Hall-Petch contribution to strength and as predicted by the theory (red symbols). The dashed lines show predictions using a line tension of one-half the original value (i.e. $\alpha$ is reduced by $1 / 2$ ), showing the weak sensitivity of the model to the line tension except at very low T; (c), Experimental [4, 5, 8] vs. predicted strengths for HEA alloys in the Ni-Co-Fe-Cr-Mn family at $T=293 K$, after subtraction of the Hall-Petch contribution to strength.

(iii) the stacking fault energy has little influence on yield strength; and (iv) local chemical environments and structural disorder should generate an additional contribution to the strength (see Eqs. (4), (15) and (16), even though such fluctuations may be difficult to measure.

The present model accounts for the strength of $N$-component random alloys at arbitrary composition, based only on solute/dislocation interaction energies. The use of average interactions provides the lowest-order approximation, and the inclusion of fluctuations in interaction energy represents the first step towards inclusion of local chemical and structural effects. Higher order effects associated with multi-solute interactions can be included within the same effective medium matrix approximation. Similarly, Short Range Order (SRO) could also be included (although SRO is only rarely observed in HEAs to date [42]). Both aspects would significantly complicate the analysis, due to statistics and correlations, but remain within the scope of the general approach here.

The present validated theory provides insights and guidance for understanding and designing strengthening in fcc HEAs. The full theory does not have any adjustable parameters; all inputs (solute/dislocation interaction energies, elastic constants, Burgers vector, dislocation core structure, line tension) can, in principle, be computed. To properly account for chemistry and magnetism, and to explore new potential alloys, $a b$ initio approaches are probably required. Currently, $C_{i j}, b, \Delta \bar{V}_{n}$, $\sigma_{\Delta V_{n}}^{2}$, and $\gamma_{\mathrm{SF}}$ can be computed by ab initio methods. The dislocation core structure, direct solute/dislocation core interactions, and the dislocation line tension, are far more challenging, and perhaps prohibitively expensive, to compute. Thus, $a b$ initio-based design is likely best pursued using the elasticity-based model (Eqs. (14)-(16) ) with dislocation structures parameterized by $b, \gamma_{\mathrm{SF}}$ and $\gamma_{\mathrm{USF}}$, and a line tension scaled by $\mu b^{2}$. Within the simplified elasticity model, higher strengths will generally be obtained for a larger solute misfit parameter $\left[\sum_{n} c_{n}\left(\Delta \bar{V}_{n}^{2}+\sigma_{\Delta V_{n}}^{2}\right)\right]^{1 / 2}$ and/or larger $\mu$, with $\gamma_{\mathrm{SF}}$ of less importance. New alloys designed using the elasticity-based theory with computable material inputs will, while not exact, provide a clear physical framework for identifying promising compositions to achieve higher-performance HEAs.

\section{Appendix A. Standard deviation of the po- tential energy change}

In this appendix, we derive the standard deviation of the potential energy change $\sigma_{\Delta U_{\text {tot }}}(\zeta, w)$ when a dislocation segment of length $\zeta$ glides over 
a distance $w$ in a random field of solutes, in a $N$ component alloy at arbitrary composition.

The interaction for a solute of type $n$ at position $\left(x_{i}, y_{j}\right)$ relative to the center of the dislocation and position $z_{k}$ along the dislocation is denoted as $U^{n}\left(x_{i}, y_{j}, z_{k}\right)$. Positions $\left(x_{i}, y_{j}, z_{k}\right)$ refer to the effective medium atomic sites, and we denote $N_{s}$ the number of atomic sites along $z$ direction for a dislocation segment of length $\zeta$, at each atomic row $\left(x_{i}, y_{j}\right)$, with $1 \leq k \leq N_{s}$. For fcc material and $\{111\}(110)$ dislocations we have $N_{s}=\zeta / \sqrt{3} b$. The total potential energy of the dislocation segment is

$U_{\text {tot }}(\zeta)=\sum_{i, j, k} \sum_{n} s_{i j k}^{n} U^{n}\left(x_{i}, y_{j}, z_{k}\right)$,

with $s_{i j k}^{n}=1$ if a type- $n$ solute is at position $\left(x_{i}, y_{j}, z_{k}\right)$, and 0 otherwise. Along the line direction $z$ of the dislocation, the dislocation structure does not change but there can be varying local chemical and structural environments that give rise to fluctuations of the interaction energy of a solute type $n$ with the dislocation along the line. Thus, for each $\left(x_{i}, y_{j}\right)$ and solute $n$, we consider a statistical distribution of interaction energies that exist along the line $z$. For ease of manipulation, we describe this distribution as a discrete distribution of $\alpha_{\max }$ different possible local environments for each solute type $n$. The corresponding solute/dislocation interaction energies are denoted as $U^{n \alpha}\left(x_{i}, y_{j}\right)$, with $1 \leq \alpha \leq \alpha_{\max }$. Different environments at different sites are assumed uncorrelated. Eq. A.1 is then rewritten as

$U_{\mathrm{tot}}(\zeta)=\sum_{i, j, k} \sum_{n, \alpha} s_{i j k}^{n \alpha} U^{n \alpha}\left(x_{i}, y_{j}\right)$,

with $s_{i j k}^{n \alpha}=1$ if a type- $n$ solute with local environment $\alpha$ sits at position $\left(x_{i}, y_{j}\right)$ and 0 otherwise. Thus, the total potential energy change when a dislocation glides a distance $w$ along the $x$ direction is

$\Delta U_{\text {tot }}(\zeta, w)=\sum_{i, j} \sum_{n, \alpha} \mathbf{n}_{i j}^{n \alpha} \Delta U_{i j}^{n \alpha}(w)$,

with

$\Delta U_{i j}^{n \alpha}(w)=\left[U^{n \alpha}\left(x_{i}-w, y_{j}\right)-U^{n \alpha}\left(x_{i}, y_{j}\right)\right]$,

and $\mathbf{n}_{i j}^{n \alpha}=\sum_{k=1}^{N_{s}} s_{i j k}^{n \alpha}$ the number of solutes $n$ with local environment $\alpha$ along dislocation line of length $\zeta$ at position $\left(x_{i}, y_{j}\right)$.
The quantity of interest in the strengthening model is the standard deviation of the total interaction energy change as defined in Eq. (2), where now both the concentrations and the interaction energies of each solute $n$ can fluctuate. We have, assuming that two solutes located in different atomic rows are uncorrelated and ommitting the dependancies in $\zeta$ and $w$ for the sake of brevity

$$
\begin{aligned}
& \sigma_{\Delta U_{\text {tot }}}^{2}=\sum_{i, j}\left[\sum_{n, \alpha}\left[\left\langle\mathbf{n}_{i j}^{n \alpha 2}\right\rangle-\left\langle\mathbf{n}_{i j}^{n \alpha}\right\rangle^{2}\right] \Delta U_{i j}^{n \alpha 2}\right. \\
& +\sum_{\substack{n \\
\alpha, \beta \neq \alpha}}\left[\left\langle\mathbf{n}_{i j}^{n \alpha} \mathbf{n}_{i j}^{n \beta}\right\rangle-\left\langle\mathbf{n}_{i j}^{n \alpha}\right\rangle\left\langle\mathbf{n}_{i j}^{n \beta}\right\rangle\right] \Delta U_{i j}^{n \alpha} \Delta U_{i j}^{n \beta} \\
& \left.+\sum_{\substack{n, m \neq n \\
\alpha, \beta}}\left[\left\langle\mathbf{n}_{i j}^{n \alpha} \mathbf{n}_{i j}^{m \beta}\right\rangle-\left\langle\mathbf{n}_{i j}^{n \alpha}\right\rangle\left\langle\mathbf{n}_{i j}^{m \beta}\right\rangle\right] \Delta U_{i j}^{n \alpha} \Delta U_{i j}^{m \beta}\right],
\end{aligned}
$$

which involves the variances and co-variances of the random variables $\left\{\mathbf{n}_{i j}^{n \alpha}\right\}$. By inspection, $\left\langle\mathbf{n}_{i j}^{n \alpha}\right\rangle=$ $p_{n \alpha} c_{n} N_{s}$, where $p_{n \alpha}$ is the probability of finding a type- $n$ solute with local environment $\alpha$, with the normalization condition $\sum_{\alpha=1}^{\alpha_{\max }} p_{n \alpha}=1$. For $(n, \alpha) \neq(m, \beta)$ we have, also by inspection,

$$
\left\langle\mathbf{n}_{i j}^{n \alpha} \mathbf{n}_{i j}^{m \beta}\right\rangle=\left(p_{n \alpha} c_{n} N_{s}\right)\left(p_{m \beta} c_{m}\left(N_{s}-1\right)\right) .
$$

The former term corresponds to the average number of type $(n, \alpha)$ solute in $N_{s}$ sites, and the latter to the average number of type $(m, \beta)$ solute in $N_{s}-1$ sites, given that at least one site is occupied by a type $(n, \alpha)$ solute. Finally, for $(n, \alpha)=(m, \beta)$ we have

$$
\begin{aligned}
\left\langle\mathbf{n}_{i j}^{n \alpha 2}\right\rangle= & \sum_{k_{n}} \frac{N_{s} !}{k_{n} !\left(N_{s}-k_{n}\right) !} c_{n}^{k_{n}} q_{n}^{N_{s}-k_{n}} \\
& \times \sum_{k_{n \alpha}} \frac{k_{n} !}{k_{n \alpha}\left(k_{n}-k_{n \alpha}\right) !} p_{n \alpha}^{k_{n \alpha}} q_{n \alpha}^{k_{n}-k_{n \alpha}} k_{n \alpha}^{2} \\
= & \sum_{k_{n}} \frac{N_{s} !}{k_{n} !\left(N_{s}-k_{n}\right) !} c_{n}^{k_{n}} q_{n}^{N_{s}-k_{n}} \\
& \times\left[p_{n \alpha}^{2} k_{n}^{2}+k_{n} p_{n \alpha}\left(1-p_{n \alpha}\right)\right] \\
= & p_{n \alpha}^{2}\left[c_{n}^{2} N_{s}^{2}+c_{n}\left(1-c_{n}\right) N_{s}\right] \\
& +p_{n \alpha}\left(1-p_{n \alpha}\right) c_{n} N_{s} \\
= & N_{s}\left[p_{n \alpha} c_{n}+p_{n \alpha}^{2} c_{n}^{2}\left(N_{s}-1\right)\right],
\end{aligned}
$$

with $0 \leq k_{n} \leq N_{s}$ and $0 \leq k_{n \alpha} \leq k_{n}$. After inser- 
This is a pre-print of the following article: Varvenne, C.; Luque, A.; Curtin, W. A. Acta Mater. 2016, 118, 164-176.. The formal publication is available at http://dx.doi.org/10.1016/j.actamat.2016.07.040

tion into Eq. A.5, we obtain

$$
\begin{aligned}
\sigma_{\Delta U_{\text {tot }}}^{2}= & N_{s} \sum_{i, j}\left[\sum_{n, \alpha} p_{n \alpha} c_{n}\left(1-p_{n \alpha} c_{n}\right) \Delta U_{i j}^{n \alpha 2}\right. \\
& -\sum_{\substack{n \neq \alpha \\
\alpha, \beta \neq \alpha}} p_{n \alpha} p_{n \beta} c_{n}^{2} \Delta U_{i j}^{n \alpha} \Delta U_{i j}^{n \beta} \\
& \left.-\sum_{\substack{n, m \neq n \\
\alpha, \beta}} p_{n \alpha} p_{m \beta} c_{n} c_{m} \Delta U_{i j}^{n \alpha} \Delta U_{i j}^{m \beta}\right] .
\end{aligned}
$$

Eq. A.8 can be simplified since $\sum_{n=1}^{N} c_{n} \sum_{\alpha=1}^{\alpha_{\max }} p_{n \alpha} \Delta U_{i j}^{n \alpha}(w)=0 \quad$ (the solute/dislocation interaction energies are defined with respect to an effective medium at the overall composition) and using the normalization of the $p_{n \alpha}$. This leads to

$$
\sigma_{\Delta U_{\mathrm{tot}}}^{2}(\zeta, w)=\frac{\zeta}{\sqrt{3} b} \sum_{i, j} \sum_{n} c_{n} \sum_{\alpha} p_{n \alpha} \Delta U_{i j}^{n \alpha}(w)^{2},
$$

using $N_{s}=\zeta / \sqrt{3} b$. Finally, writing each specific class of interaction energy variation for a type $n$ solute as a deviation from the average value, i.e. $\Delta U_{i j}^{n \alpha}(w)=\Delta \bar{U}_{i j}^{n}(w)+\delta U_{i j}^{n \alpha}(w)$, we obtain (without assuming that deviations are small)

$\sigma_{\Delta U_{\mathrm{tot}}}^{2}(\zeta, w)=\frac{\zeta}{\sqrt{3} b} \sum_{i, j} \sum_{n} c_{n}\left(\Delta \bar{U}_{i j}^{n}(w)^{2}+\sigma_{\Delta U_{i j}^{n}}^{2}\right)$,

with $\sigma_{\Delta U_{i j}^{n}}^{2}=\sum_{\alpha} p_{n \alpha} \delta U_{i j}^{n \alpha 2}$ being the square of the standard deviation of the distribution of the interaction energy change $\left\{\Delta U_{i j}^{n \alpha}(w)\right\}$. Interestingly, even in the case of a zero average interaction energy, a type- $n$ solute can still contribute in the strengthening due to the fluctuations of the interaction energy, which appear through the standard deviation in Eq. A.10. One specific case where this applies is for the interaction energy between local deviatoric strains of solutes $n$ and the deviatoric stress field of the dislocation, where the average deviatoric strains must be zero by symmetry for substitutional solutes in fcc materials.

\section{Appendix B. Connection to the $\delta$ parameter}

Neglecting the standard deviation term in Eq. A.10, we can further connect $\sigma_{\Delta U_{\text {tot }}}(\zeta, w)$ to the so-called misfit parameter [2, 3, 43, 44. This connection arises through the "solute" quantity $\sum_{n} c_{n} \Delta \bar{V}_{n}^{2}$ that emerges in the present theory. The average misfit volume $\Delta \bar{V}_{n}$ is related to the variation in volume with respect to alloy composition as

$\Delta \bar{V}_{n}=\sum_{m} c_{m}\left[\left.\frac{\partial \bar{V}}{\partial c_{n}}\right|_{\bar{c}}-\left.\frac{\partial \bar{V}}{\partial c_{m}}\right|_{\bar{c}}\right]$,

with $\bar{V}$ the average atomic volume and where $\bar{c}=$ $\left(c_{1}, \ldots, c_{N}\right)$ refers to the composition of the $N$ component alloy of interest. For an fcc alloy, we have $\bar{V}=a^{3} / 4$, with $a$ the lattice parameter of the alloy, and thus

$\Delta \bar{V}_{n}=\frac{3 \bar{V}}{a} \sum_{m} c_{m}\left[\left.\frac{\partial a}{\partial c_{n}}\right|_{\bar{c}}-\left.\frac{\partial a}{\partial c_{m}}\right|_{\bar{c}}\right]$.

If we apply Vegard's law to the lattice parameter of the solid solution, i.e. $a=\sum_{n} c_{n} a_{n}$ with $a_{n}$ the lattice parameter of element $n$, then

$\Delta \bar{V}_{n}=3 \bar{V}\left(\frac{a_{n}}{a}-1\right)$.

The key solute quantity in the strengthening theory is thus

$\sum_{n} c_{n} \Delta \bar{V}_{n}^{2}=9 \bar{V}^{2}\left[\frac{\sum_{n} c_{n} r_{n}^{2}}{r^{2}}-1\right]=9 \bar{V}^{2} \delta^{2}$,

where $r_{n}$ and $r$ are the atomic radii of the element $n$ and of an average atom, respectively. We thus obtain a direct connection with the misfit parameter $\delta$ used to qualitatively characterize HEAs [2, 3]. However, $\delta$ is usually estimated using tables of "accepted" atomic radii for the elements. For robust application of the strengthening theory, it is much better to use the true solute misfit volumes in the actual alloy of interest, which are well-defined quantities from both thermodynamics and mechanics perspectives, and do not rely on the validity of Vegard's law, nor require an assumption that the different elements crystallize into the same structure.

\section{Appendix C. Atomistic details about MS shear tests}

Atomistic measurements of the flow stresses for $\mathrm{Fe}_{(1-x) / 2} \mathrm{Ni}_{(1-x) / 2} \mathrm{Cr}_{x}$ EAM alloys are performed using the open-source code LAmmPs [45]. For each 
This is a pre-print of the following article: Varvenne, C.; Luque, A.; Curtin, W. A. Acta Mater. 2016, 118, 164-176.. The formal publication is available at http://dx.doi.org/10.1016/j.actamat.2016.07.040

composition $x, 20$ different random realizations at the overall composition are prepared. In each of them, a straight dislocation is introduced as two Shockley partials separated by the proper dissociation distance using the Volterra displacement field. The system is relaxed to its local minimum energy state, with traction free boundaries on the surfaces of the sample parallel to the glide plane, and periodic boundary conditions in both the glide and line directions [46]. The position of the dislocation is continuously monitored as the average of the positions of the two partial dislocations. The simulation cell sizes are $X \times Y \times Z=100 b \times \zeta_{c} \times 48 b$, with $X$ the glide direction, $Y$ the line direction and $Z$ the (111) direction, thus large enough to prevent any finite-size effects.

We then apply an increasing resolved shear stress, with steps of $5 \mathrm{MPa}$. We start from $\tau_{0}=250 \mathrm{MPa}$ for $x=0.1$, from $200 \mathrm{MPa}$ for $x=0.2$ and 0.33 , from $150 \mathrm{MPa}$ for $x=0.4$, and from $100 \mathrm{MPa}$ for $x=0.5$. Some samples having initial dislocation jumps $x_{d}>w_{c} / 2$ were re-started with lower values for the applied shear stress, so as to accurately describe the behavior around the dislocation position $x_{\mathrm{d}}=w_{c} / 2$. Results are displayed in Fig. C.8 for the five EAM alloys.

We then computed the average shear stress $\tau$ vs. dislocation position by using a grid of point $x_{i}=i b / 2$, with $i=1,2, \ldots$ The window around each grid point $x_{i}$ is $\pm b / 4$, consistent with the precision in the theory: $w_{c} \propto b / 2$. We set $\tau\left(x_{\mathrm{d}}=0\right)=0$, and the average shear stress for dislocation positions in $\left[0, b / 4\left[\right.\right.$ is centered at $x_{0}=b / 8$. The average curves are shown in black in Fig. C.8 In the $x=0.1,0.4$ and $0.5 \mathrm{Fe}_{(1-x) / 2} \mathrm{Ni}_{(1-x) / 2} \mathrm{Cr}_{x}$ alloys, for the small values of $x_{i}$, typically $b / 8$ and $b / 2$, some samples already experience a big displacement jump at the initial stress $\tau_{0}$, i.e. the shear stress required to move the dislocation is smaller than $\tau_{0}$ within that interval. In evaluating the average strength, we thus fixed for these samples the stress value to $\tau_{0} / 2$, so as to obtain a reasonable estimate of $\tau$ (taking only the other available data points would lead to an overestimation of $\tau$ ). The less accurate sampling for the average stress is reflected in the associated $70 \%$ confidence intervals in Figs. 5a,b, which are then higher for the low $x_{i}$ values. For the $x=0.2$ and 0.33 alloys, we exclude the $[0, b / 4[$ zone for the average strength curve, as the sampling is not sufficient to define an average.

The theory assumes a Gaussian distribution for the fluctuations in potential energy, with the key

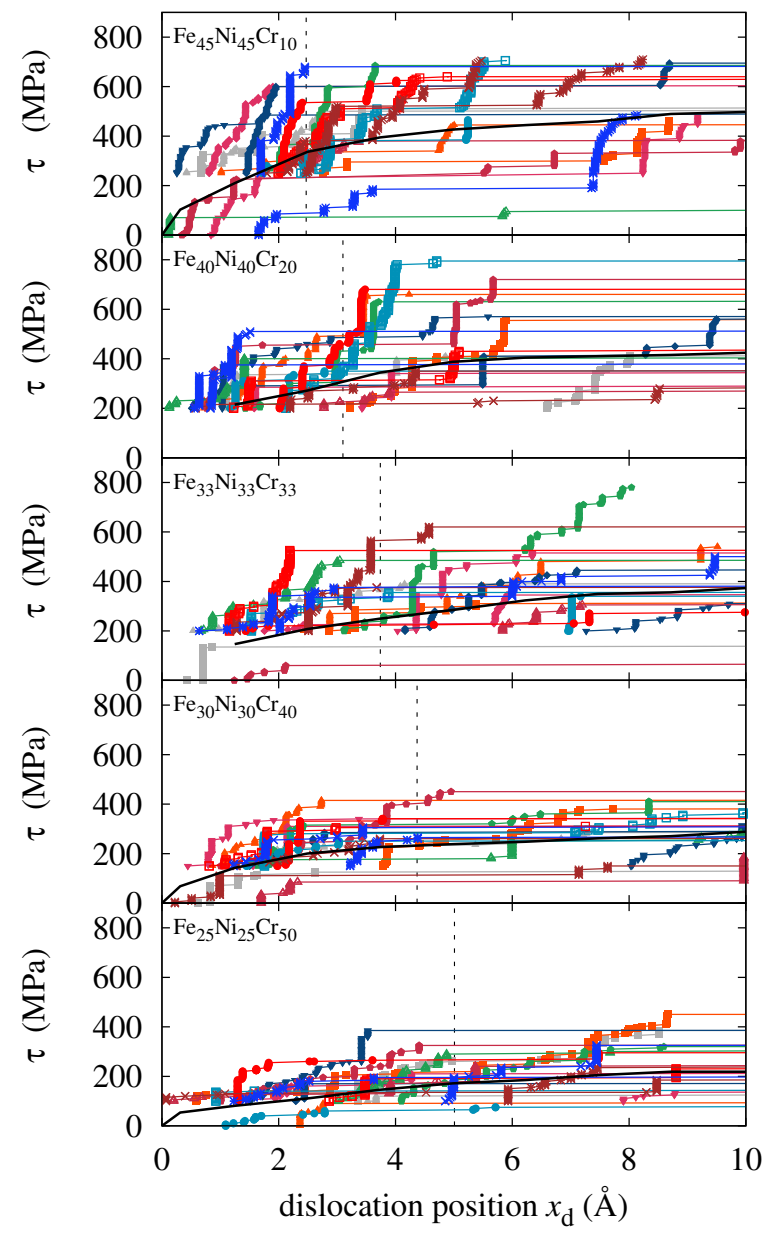

Figure C.8: Molecular Statics simulations of the flow stress for $\mathrm{Fe}_{(1-x) / 2} \mathrm{Ni}_{(1-x) / 2} \mathrm{Cr}_{x}$ EAM alloys. The 20 different samples for each targeted composition $x$ are shown by the colored symbols and markers. The average applied stress $\tau$ vs. average dislocation position $x_{\mathrm{d}}$ is shown as the black line. The vertical dashed line indicates the value of $w_{c} / 2$ predicted by the theory as the point at which the dislocation becomes unpinned, on average.

energy quantity $\Delta \tilde{E}_{p}\left(w_{c}\right)$ related to the standard deviation of the interaction energy $\sigma_{\Delta U_{\text {tot }}}$, which corresponds to the average for pinning in the lower $33 \%$ of the probability distribution function. Variations around this average strength are expected in simulations from the theory, and a large spreading in strength is indeed observed, reflecting the underlying statistical nature of the stengthening. The simulations also reveal the underlying length scale $w_{c}$ that the theory predicts (Fig. C.8 dashed lines) and its evolution with the overall alloy composition: most of the samples undergo a significant displace- 
This is a pre-print of the following article: Varvenne, C.; Luque, A.; Curtin, W. A. Acta Mater. 2016, 118, 164-176.. The formal publication is available at http://dx.doi.org/10.1016/j.actamat.2016.07.040

ment jump when $x \simeq w_{c} / 2$.

\section{Appendix D. Determination of the disloca- tion partial spreading}

We parameterize the Burgers vector distribution of the dissociated dislocation using two Gaussian peaks separated by a distance $d$ and with standard deviation of each Gaussian denoted by $\sigma$. The Burgers vector distribution is divided into a discrete set of fractional Burgers vector on the discrete lattice points

$$
\frac{\Delta b\left(x_{i}\right)}{b}=\frac{e^{-\frac{1}{2}\left(\frac{x_{i}-d / 2}{\sigma}\right)^{2}}+e^{-\frac{1}{2}\left(\frac{x_{i}+d / 2}{\sigma}\right)^{2}}}{\sum_{k=-\infty}^{+\infty} e^{-\frac{1}{2}\left(\frac{x_{k}-d / 2}{\sigma}\right)^{2}}+e^{-\frac{1}{2}\left(\frac{x_{k}+d / 2}{\sigma}\right)^{2}}},
$$

where $x_{i}=n b / 2$ with $n=0, \pm 1, \pm 2 \ldots$ is the position along the glide plane, $b$ is the Burgers vector of the edge perfect dislocation, and where $d$ is the partial separation distance. Isotropic elasticity predicts $d=\frac{\mu b^{2}}{\gamma_{\mathrm{SF}}} \frac{(2+\nu)}{24 \pi(1-\nu)}$ for an fcc edge dislocation, with $\mu, \nu$ the elastic constants and $\gamma_{\mathrm{SF}}$ the stable stacking fault energy.

The spreading within the partial cores is represented by the parameter $\sigma$. In a Peierls-Nabarro model for the core [4], the spreading of the partial cores should scale with $\mu b^{2} /\left(4 \pi(1-\nu) \gamma_{\mathrm{USF}}\right)$, where $\gamma_{\text {USF }}$ is the unstable stacking fault energy. The precise scaling depends on details of the entire generalized stacking fault curve, and this is not available for the HEA materials. To determine a good value for $\sigma$, we consider two different analysis.

First, we study the core structures in the random $\mathrm{Fe}_{(1-x) / 2} \mathrm{Ni}_{(1-x) / 2} \mathrm{Cr}_{x}$ EAM alloys with $x=10,20$, 33,40 and $50 \%$, having different material parameters. Employing the effective medium approach for EAM potentials 22, we relax edge dislocations in the effective medium for each alloy and fit the resulting Burgers vector distributions to Eq. (D.1) to obtain $\sigma$ values for all alloys. Fig. D.9a,b show the measured $\sigma$ values and the same values normalized by $\mu b^{2} /\left(4 \pi(1-\nu) \gamma_{\mathrm{USF}}\right)$, vs. Cr content $x$. The variations in $\sigma$ are small among the different materials, ranging from $1.5 b$ to $2.5 b$, and the near-constant normalized value verifies the expected scaling. Using this constant value of $\sim 0.28$ in Fig. D.9b for pure $\mathrm{Ni}\left(\mu=79 \mathrm{GPa}, \gamma_{\mathrm{USF}}=113 \mathrm{~mJ} / \mathrm{m}^{2}\right)$ and for pure $\mathrm{Al}\left(\mu=28.4 \mathrm{GPa}, \gamma_{\mathrm{USF}}=224 \mathrm{~mJ} / \mathrm{m}^{2}\right)$, we obtain $\sigma=1.74 b$ and $1.26 b$, respectively (materials parameters from Refs. 33 and 48 for $\mathrm{Ni}$ and $\mathrm{Al}$ ).

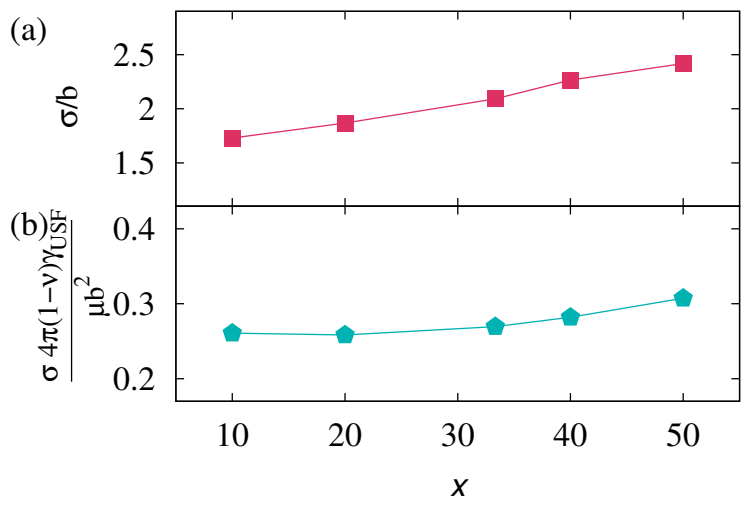

Figure D.9: (a), Partial core spreading $\sigma$ in $\mathrm{Fe}_{(1-x) / 2} \mathrm{Ni}_{(1-x) / 2} \mathrm{Cr}_{x}$ EAM alloys, measured by fitting the atomistically-determined Burgers vector distributions with the distribution of Eq. (D.1). (b), Dimensionless ratio of the measured $\sigma$ to the material quantity $\mu b^{2} /\left(4 \pi(1-\nu) \gamma_{\mathrm{USF}}\right)$ for the various alloys, showing a constant scaling as expected from a Peierls-Nabarro model [47.

Thus, the value of $\sigma=1.5 b$ we used for HEAs is generally valid for these fcc systems.

Second, we consider the dilute solutestrengthening predictions of Leyson et al. [27] for six substitutional solutes $(\mathrm{Mg}, \mathrm{Cu}, \mathrm{Si}, \mathrm{Cr}, \mathrm{Mn}$, $\mathrm{Fe}$ ) in $\mathrm{Al}$. In these systems, full DFT calculations of the solute/dislocation core interactions and solute misfit volumes were performed. Using only the misfit volume interaction we make predictions of $\tau_{y 0}$ as a function of core structure, using dissociation distances $d=2.5 b, 3 b$ consistent with the DFT-computed core [49] and varying partial core spreading $\sigma$. All other Al parameters are given in Ref. 27. The best agreement with the full DFT-computed results is obtained for $\sigma=1.5 b$, justifying again our choice of $\sigma=1.5 b$ as a fixed parameter for the dislocation partial core spreading in the HEA alloys.

\section{Acknowledgments}

Support for this work was provided through a European Research Council Advanced Grant, "Predictive Computational Metallurgy", ERC Grant agreement No. 339081 - PreCoMet. Dr. F. Pavia is acknowledged for performing the atomistic line tension measurements. 
This is a pre-print of the following article: Varvenne, C.; Luque, A.; Curtin, W. A. Acta Mater. 2016, 118, 164-176.. The formal publication is available at http://dx.doi.org/10.1016/j.actamat.2016.07.040

\section{References}

[1] M.-H. Tsai, J.-W. Yeh, High-entropy alloys: A critical review, Mater. Res. Lett. 2 (3) (2014) 107-123. doi: $10.1080 / 21663831.2014 .912690$

[2] Y. Zhang, T. T. Zuo, Z. Tang, M. C. Gao, K. A. Dahmen, P. K. Liaw, Z. P. Lu, Microstructures and properties of high-entropy alloys, Prog. Mater. Sci. 61 (2014) 1-93. doi:10.1016/j.pmatsci.2013.10.001.

[3] D. B. Miracle, J. D. Miller, O. N. Senkov, C. Woodward, M. D. Uchic, J. Tiley, Exploration and development of high entropy alloys for structural applications, Entropy 16 (1) (2014) 494-525. doi:10.3390/e16010494

[4] Z. Wu, H. Bei, G. Pharr, E. George, Temperature dependence of the mechanical properties of equiatomic solid solution alloys with face-centered cubic crystal structures, Acta Mater. 81 (0) (2014) 428 - 441. doi: http://dx.doi.org/10.1016/j.actamat.2014.08.026

[5] Z. Wu, H. Bei, F. Otto, G. Pharr, E. George, Recovery, recrystallization, grain growth and phase stability of a family of fcc-structured multi-component equiatomic solid solution alloys, Intermetallics 46 (0) (2014) 131 - 140. doi:http://dx.doi.org/10.1016/j.intermet. 2013.10.024

[6] B. Gludovatz, A. Hohenwarter, D. Catoor, E. H. Chang, E. P. George, R. O. Ritchie, A fracture-resistant high-entropy alloy for cryogenic applications, Science 345 (6201) (2014) 1153-1158. doi:10.1126/science. 1254581

[7] M. Poletti, L. Battezzati, Electronic and thermodynamic criteria for the occurrence of high entropy alloys in metallic systems, Acta Mater. 75 (2014) 297 - 306. doi:http://dx.doi.org/10.1016/j.actamat. 2014.04 .033

[8] F. Otto, A. Dlouhý, C. Somsen, H. Bei, G. Eggeler, E. George, The influences of temperature and microstructure on the tensile properties of a cocrfemnni high-entropy alloy, Acta Mater. 61 (15) (2013) 5743 - 5755. doi:http://dx.doi.org/10.1016/j.actamat. 2013.06 .018

[9] R. Labusch, A statistical theory of solid solution hardening, Phys. Status Solidi A 41 (2) (1970) 659. doi: $10.1002 / \mathrm{pssb} .19700410221$

[10] M. Zaiser, Dislocation motion in a random solid solution, Philos. Mag. A 82 (15) (2002) 2869-2883. doi: doi: $10.1080 / 01418610208240071$

[11] A. Argon, Strengthening Mechanisms in Crystal Plasticity, Oxford Univ. Press, 2007.

[12] G. P. M. Leyson, W. A. Curtin, L. G. Hector, Jr., C. F. Woodward, Quantitative prediction of solute strengthening in aluminium alloys, Nat. Mater. 9 (9) (2010) 750-755. doi:10.1038/NMAT2813.

[13] J. A. Yasi, L. G. Hector Jr, D. R. Trinkle, Firstprinciples data for solid-solution strengthening of magnesium: From geometry and chemistry to properties, Acta Mater. 58 (17) (2010) 5704 - 5713. doi:http: //dx.doi.org/10.1016/j.actamat.2010.06.045

[14] D. Ma, M. Friák, J. von Pezold, D. Raabe, J. Neugebauer, Computationally efficient and quantitatively accurate multiscale simulation of solid-solution strengthening by ab initio calculation, Acta Mater. 85 (2015) 53 - 66. doi:http://dx.doi.org/10.1016/j.actamat. 2014.10 .044

[15] R. J. Elliott, J. A. Krumhansl, P. L. Leath, The theory and properties of randomly disordered crystals and related physical systems, Rev. Mod. Phys. 46 (1974) 465-543. doi:10.1103/RevModPhys.46.465

[16] J. Faulkner, The modern theory of alloys, Prog. Mater. Sci.s 27 (1-2) (1982) 1 - 187. doi:http://dx.doi.org/ 10.1016/0079-6425(82) 90005-6

[17] D. D. Johnson, D. M. Nicholson, F. J. Pinski, B. L. Gyorffy, G. M. Stocks, Density-functional theory for random alloys: Total energy within the coherent-potential approximation, Phys. Rev. Lett. 56 (1986) 2088-2091. doi:10.1103/PhysRevLett.56.2088

[18] F. Ducastelle, Order and Phase Stability in Alloys, North-Holland, Amsterdam, 1991.

[19] D. Johnson, M. Asta, Energetics of homogeneouslyrandom fcc al-ag alloys: A detailed comparison of computational methods, Comput. Mater. Sci. 8 (1-2) (1997) $54-63$, proceedings of the joint NSF/CNRS Workshop on Alloy Theory. doi:http://dx.doi.org/10. 1016/S0927-0256(97)00016-5

[20] B. Ujfalussy, J. S. Faulkner, N. Y. Moghadam, G. M. Stocks, Y. Wang, Calculating properties with the polymorphous coherent-potential approximation, Phys. Rev. B 61 (2000) 12005-12016. doi:10.1103/PhysRevB. 61.12005

[21] R. W. Smith, G. S. Was, Application of molecular dynamics to the study of hydrogen embrittlement in ni-cr-fe alloys, Phys. Rev. B 40 (1989) 10322-10336. doi:10.1103/PhysRevB.40.10322

[22] C. Varvenne, A. Luque, W. G. Nöhring, W. A. Curtin, Average-atom interatomic potentials for random alloys, accepted to Phys. Rev. B.

[23] S. Rao, C. Woodward, T. A. Parthasarathy, D. Dimiduk, D. B. Miracle, W. A. Curtin, Molecular statics and molecular dynamics simulations of dislocation behavior in model fcc and bcc multicomponent concentrated solid solution alloys, in preparation.

[24] D. J. Bacon, D. M. Barnett, R. O. Scattergood, Anisotropic continuum theory of lattice defects, Prog. Mater. Sci. 23 (1980) 51-262. doi:10.1016/ 0079-6425(80)90007-9

[25] E. Clouet, The vacancy - edge dislocation interaction in fcc metals, in: P. Gumbsch (Ed.), Proceedings of the Third International Conference Multiscale Materials Modeling, Freiburg, Germany, 2006, pp. 708-711.

[26] E. Hayward, C. Deo, B. P. Uberuaga, C. N. Tomé, The interaction of a screw dislocation with point defects in bcc iron, Phil. Mag. 92 (2012) 2759. doi: 10.1080/14786435.2012.674646

[27] G. P. M. Leyson, L. Hector Jr., W. A. Curtin, Solute strengthening from first principles and application to aluminum alloys, Acta Mater. 60 (9) (2012) 3873 - 3884. doi:http://dx.doi.org/10.1016/j.actamat. 2012.03 .037

[28] G. P. M. Leyson, L. Hector Jr., W. A. Curtin, Firstprinciples prediction of yield stress for basal slip in mg-al alloys, Acta Mater. 60 (13-14) (2012) 5197 - 5203. doi:http://dx.doi.org/10.1016/j.actamat. 2012.06 .020

[29] U. Kocks, A. S. Argon, M. F. Ashby, Prog. Mater. Sci. 19 (1975) 1 - 281. doi:http://dx.doi.org/10.1016/ 0079-6425(75) 90005-5

[30] R. Labusch, G. Grange, J. Ahearn, P. Haasen, Rate processes in plastic deformation of materials, in: J. C. M. Li, A. K. Mukherjee (Eds.), Proceedings from the John E. Dorn symposium, ASM, Metal Park, Ohio, 1975, p. 26. 
This is a pre-print of the following article: Varvenne, C.; Luque, A.; Curtin, W. A. Acta Mater. 2016, 118, 164-176.. The formal publication is available at http://dx.doi.org/10.1016/j.actamat.2016.07.040

[31] G. P. M. Leyson, W. A. Curtin, Solute strengthening at high temperatures, submitted.

[32] R. Labusch, Cooperative effects in alloy hardening, Czech. J. Phys. 38 (5) (1988) 474-481. doi:10.1007/ BF01597457

[33] G. Bonny, D. Terentyev, R. C. Pasianot, S. Ponce, A. Bakaev, Interatomic potential to study plasticity in stainless steels: the fenicr model alloy, Modelling Simul. Mater. Sci. Eng. 19 (8). doi:10.1088/0965-0393/19/8/ 085008

[34] J. P. Hirth, J. Lothe, Theory of Dislocations, 2nd Edition, Wiley, New York, 1982.

[35] A. J. Zaddach, C. Niu, C. C. Koch, D. L. Irving, Mechanical properties and stacking fault energies of nifecrcomn high-entropy alloy, JOM 65 (12) (2013) 17801789. doi:10.1007/s11837-013-0771-4

[36] A. Haglund, M. Koehler, D. Catoor, E. George, V. Keppens, Polycrystalline elastic moduli of a high-entropy alloy at cryogenic temperatures, Intermetallics $58(0)$ (2015) 62 - 64. doi:http://dx.doi.org/10.1016/j. intermet.2014.11.005

[37] G. Laplanche, P. Gadaud, O. Horst, F. Otto, G. Eggeler, E. George, Temperature dependencies of the elastic moduli and thermal expansion coefficient of an equiatomic, single-phase cocrfemnni high-entropy alloy, J. Alloys Compd. 623 (0) (2015) 348 - 353. doi: http://dx.doi.org/10.1016/j.jallcom.2014.11.061.

[38] J. Bandyopadhyay, K. P. Gupta, Low temperature lattice parameter of nickel and some nickel-cobalt alloys and grüneisen parameter of nickel, Cryogenics (1977) 345-347.

[39] F. Abe, T. Tanabe, Change in lattice spacing of nickel by dissolved chromium and tungsten, Z. Metall. 76 (6) (1985) 420-425.

[40] N. J. D. Tilley, Understanding Solids: The Science of Materials, Wiley, 2004.

[41] B. Szajewski, F. Pavia, Y. Dong, W. A. Curtin, Robust line tension via atomistics, Modelling Simul. Mater. Sci. Eng. 23 (8).

[42] C. Niu, A. J. Zaddach, A. A. Oni, X. Sang, J. W Hurt, J. M. LeBeau, C. C. Koch, D. L. Irving, Spindriven ordering of $\mathrm{cr}$ in the equiatomic high entropy alloy nifecrco, Appl. Phys. Lett. 106 (16). doi:http: //dx.doi.org/10.1063/1.4918996

[43] I. Toda-Caraballo, P. E. J. Rivera-Díaz-del Castillo, Modelling solid solution hardening in high entropy alloys, Acta Mater. 85 (0) (2015) 14 - 23. doi:http: //dx.doi.org/10.1016/j.actamat.2014.11.014

[44] X. Li, H. Zhang, S. Lu, W. Li, J. Zhao, B. Johansson, L. Vitos, Elastic properties of vanadium-based alloys from first-principles theory, Phys. Rev. B 86 (2012) 014105. doi:10.1103/PhysRevB.86.014105

[45] S. Plimpton, Fast parallel algorithms for short-range molecular dynamics, J. Comput. Phys. 117 (1) (1995) 1 - 19. doi:http://dx.doi.org/10.1006/jcph.1995. 1039

[46] Y. N. Osetsky, D. J. Bacon, An atomic-level model for studying the dynamics of edge dislocations in metals, Modelling Simul. Mater. Sci. Eng. 11 (2003) 427-446. doi: 10.1088/0965-0393/11/4/302

[47] V. V. Bulatov, W. Cai, Computer Simulations of Dislocations, Oxford series on materials modelling, Oxford University Press, 2006.

[48] G. Lu, N. Kioussis, V. V. Bulatov, E. Kaxiras, Generalized-stacking-fault energy surface and disloca- tion properties of aluminum, Phys. Rev. B 62 (2000) 3099-3108. doi:10.1103/PhysRevB.62.3099

[49] S. M. Keralavarma, A. F. Bower, W. A. Curtin, Quantum-to-continuum prediction of ductility loss in aluminium-magnesium alloys due to dynamic strain aging, Nat. Commun. 5. doi:10.1038/ncomms5604 